\title{
THE ACTIVATION ENERGY FOR DISLOCATION NUCLEATION AT A CRACK
}

\author{
JAMES R. RiCE† and GLenN E. Beltz $\ddagger$ \\ $\dagger$ Division of Applied Sciences and Department of Earth and Planetary Sciences, Harvard University, \\ Cambridge, MA 02138, U.S.A. ; and $\ddagger$ Department of Mechanical and Environmental Engineering, \\ University of California, Santa Barbara, CA 93106, U.S.A.
}

(Received 12 June 1993)

\begin{abstract}
THE ACTIVATION energy for dislocation nucleation from a stressed crack tip is calculated within the Peierls framework, in which a periodic shear stress vs displacement relation is assumed to hold on a slip plane emanating from the crack tip. Previous results have revealed that the critical $G$ (energy release rate corresponding to the "screened" crack tip stress field) for dislocation nucleation scales with $\gamma_{\mathrm{us}}$ (the unstable stacking energy), in an analysis which neglects any coupling between tension and shear along the slip plane. That analysis represents instantaneous nucleation and takes thermal effects into account only via the weak temperature dependence of the elastic constants. In this work, the energy required to thermally activate a stable, incipient dislocation into its unstable "saddle-point" configuration is directly calculated for loads less than that critical value. We do so only with the simplest case, for which the slip plane is a prolongation of the crack planc. A first calculation reported is $2 \mathrm{D}$ in nature, and hence reveals an activation energy per unit length. A more realistic scheme for thermal activation involves the emission of a dislocation loop, an inherently $3 \mathrm{D}$ phenomenon. Asymptotic calculations of the activation energy for loads close to the critical load are performed in 2D and in 3D. It is found that the 3D activation energy generally corresponds to the 2D activation energy per unit length multiplied by about 5-10 Burgers vectors (but by as many as 17 very near to the critical loading). Implications for the emission of dislocations in copper, $\alpha$-iron, and silicon at elevated temperature are discussed. The effects of thermal activation are very significant in lowering the load for emission. Also, the appropriate activation encrgy to correspond to molccular dynamies simulations of crack tips is discussed. Such simulations, as typically carried out with only a few atomic planes in a periodic repeat direction parallel to the crack tip, are shown to greatly exaggerate the (already large) effects of temperature on dislocation nucleation.
\end{abstract}

\section{INTRODUCTION}

WE BUILD on recent advances in the modeling of dislocation nucleation at a crack tip based on the Peierls-Nabarro concept (RICE, 1992; BeLTZ and RICE, 1991, 1992; RICE et al., 1992; BELTZ, 1992; SUN et al., 1991, 1993; Sun, 1993). These have provided a consistent description of the genesis of a dislocation, free of core cut-off parameters of earlier approaches, and have predicted a critical load for emission in various materials. The analyses presented thus far, however, have neglected thermal effects, except perhaps through the weak temperature dependence of the elastic constants which enter the analysis. They thus correspond to instantaneous emission without the aid of thermal fluctuations. The purpose of this paper is to extend the Peierls model to calculate the activation energy associated with the nucleation of a 
dislocation, thereby gaining insights on the effects of elevated temperatures. The basic premise is that, assuming a crack is loaded below the critical load for instantaneous emission of a dislocation, a dislocation could nevertheless be emitted if thermal activation supplies enough energy to overcome the predicted activation energy barrier. Realistically, this is a very localized process, involving the unstable emission of a dislocation loop, and hence the problem is inherently 3D. Initially, a 2D activation energy (per unit length of dislocation line) for dislocation nucleation within the Peierls framework is calculated directly and then via an asymptotic analysis (good for loadings near the critical loading for emission). Next the asymptotic analysis is extended to the 3D situation, so as to approximately calculate the activation energy and shape of the activated slip configuration.

Rice and THOMSON (1974) regarded the ductile vs brittle behavior of materials as a competition between dislocation emission at an atomistically sharp crack and cleavage decohesion. In their work, the activation energy was calculated for a crack which had been subjected to the Griffith load for cleavage (i.e. when $G$, the "applied" energy release rate, attained the value $2 \xi_{s}$, the ideal work of fracture defined as twice the surface energy). A disadvantage is that their analysis considered a pre-existing, fullyformed dislocation on a slip plane intersecting the crack tip and hence relied on the uncertain core-cutoff parameter $r_{0}$ from elastic dislocation theory [see Fig. $1(a)$ ]; that and other ad hoc energy cut-offs, especially at the near-atomic length scales involved. provided what are now regarded as very questionable estimates of the activation

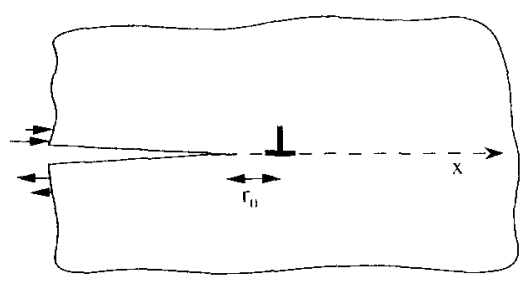

(a)

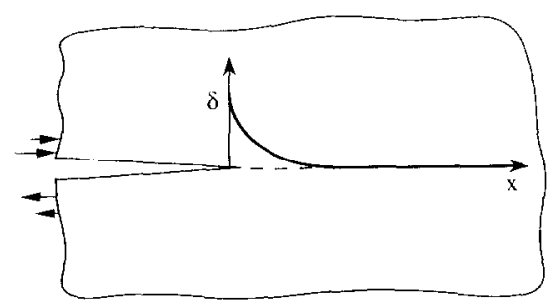

(b)

FIG. 1. Atomically sharp crack loaded in mode II, with a slip plane continuing along the crack plane. (a) Depicts a fully formed Volterra dislocation, as analyzed in the Rice Thomson model, and (b) denotes the incipient dislocation modelled as a Peierls-type shear profile. The crack surfaces are assumed to be free of tractions. 
energy (ARGON, 1987). With this limitation in mind, various crystals were classified as ductile or brittle based on the outcome of this calculation. In general, dislocations in fec metals were found to become unstable when the fully formed dislocation was within one core radius of the crack tip, and since the elastic forces considered in this calculation are ill-defined on the length scales considered, it was argued that dislocations were spontaneously emitted in these materials. The bcc metals, as well as the covalent and ionic crystals considered were found to have positive activation energies with an activated dislocation loop that was sensibly large. Examples include $\mathrm{Fe}$, with an activation energy of $2.2 \mathrm{eV}$ (which is small enough for thermal activation to be important, as will be discussed later), and $\mathrm{Si}$, with an activation energy of 111 $\mathrm{eV}$ (which is prohibitively high for thermal activation to occur at room temperature), but which is much higher than what is found here.

Through extensions by MASON (1979), ANDFrson (1986), and ANDFrson and RicE (1986), the Rice-Thomson model evolved to a state where the competition between dislocation emission and cleavage was evaluated not in terms of an activation energy but rather via the separate calculation of $G_{\text {crii }}$, the energy release rate associated with the emission of a single dislocation and $G_{\text {clcave }}$, the Griffith cleavage energy. The former depends not just on the geometry of the slip system relative to the fracture plane, but also on the ratios of stress intensity factors (modes I, II and III) to one another. If $G_{\text {crii }}<G_{\text {cleave, }}$ then it is assumed that a dislocation would be spontaneously emitted before decohesion, and vice versa. The calculation of the activation energy for dislocation emission has been additionally considered within the Rice-Thomson framework by Li $(1981,1986)$ who calculated the activation energy as a function of the applied energy release rate, rather than evaluating it at the Griffith cleavage level. Li's result, valid for a mode II load and a slip plane coplanar with the crack plane, gives the activation energy (per unit dislocation length) to emit an edge dislocation and can be written as

$$
\frac{(1-v) \Delta U_{\mathrm{acl}}}{\mu b^{2}}=\frac{-1}{4 \pi} \ln \left(\frac{G}{G_{\text {crit }}}\right),
$$

where $\mu$ and $v$ are the shear modulus and Poisson's ratio, respectively. This result will be seen to overestimate the more exact $2 \mathrm{D}$ results calculated here.

Calculations of the activation energy for dislocation emission in silicon have been of interest recently because this material undergoes a relatively sharp brittle-to-ductile transition at a temperature dependent on loading rate, e.g. at approximately $562 \mathrm{C}$ at a strain rate of $1.3 \times 10^{-6} \mathrm{~s} \cdot 1$ [see experimental work by SAMUFLS and RoBrRTS (1989), Chiao and Clarke (1989), Warren (1989), Michot and George (1986), and references therein]. Dislocation motion below the transition is not observed, and the transition temperature increases with strain rate; increasing the strain rate by a factor of 20 increases the transition temperature by about $100 \mathrm{C}$. As mentioned before, however, the Rice-Thomson estimate of the activation energy at the fracture stress for $\mathrm{Si}$ was $111 \mathrm{eV}$, making thermally activated dislocation nucleation at the transition temperature impossible. HAASEN (1983) has estimated the activation energy in $\mathrm{Si}$ at the Griffith load to be as low as $0.5 \mathrm{eV}$, taking into account the fact that the stacking fault energy in $\mathrm{Si}$ is relatively low and hence nucleation of a partial dislocation 
with Burgers vector $\mathbf{b}=(a / 6)[112]$ is favored. Additionally, I.-H. Lin and R. Thomson (private communication, 1992) have carried out a calculation of the activation energy for a rectangular partial dislocation loop in Si and obtain values between 0.5 and 1.0 $\mathrm{eV}$, making thermal activation possible; the difference between these numbers and the one calculated by Rice and Thomson is attributable to the fact that a partial dislocation is considered, and a much different saddle-point configuration was assumed (rectangular vs circular). The aforementioned numbers seem too low to account for the ductile-to-brittle transition in silicon, especially in light of recent atomic calculations by Duesbery et al. (1991) and Kaxiras and Duesbery (1993), which reveal anomalously large values of the unstable stacking energy $\gamma_{\text {us }}(=1.91$ $\mathrm{J} / \mathrm{m}^{2}$ for relaxed shear, commonly referred to as $\gamma_{\mathrm{us}}^{(\mathrm{r})}$, on the so-called "glide-set" and $1.67 \mathrm{~J} / \mathrm{m}^{2}$ on the "shuffle" set), the parameter which controls dislocation nucleation within the Peierls framework (RICE, 1992) and which will be discussed in further detail below. It has become increasingly evident in recent years that it may be lattice friction against motion of dislocations, not the nucleation event itself, which controls the brittle-to-ductile transition of silicon (MiCHOT and GEORGE, 1986; BREDE and HAASEN, 1988; Chiao and Clarke, 1989; Warren, 1989; Hirsch et al., 1989; Hirsch and ROBERTS, 1991; HSIA and ArgON, 1993). However, the issue remains incompletely resolved. Further discussion of the instantaneous nucleation of dislocations in Si may be found in recent work by Sun et al. (1993) based on parameters for Peierls modeling of Si from Kaxiras and Duesbery (1993) and HuAng et al. (1991); additionally, the detailed application of the ideas presented in this paper to the understanding of thermal activation in the brittle-to-ductile transition in silicon is the subject of a follow-up paper (BELTZ and RICE, 1994).

The most serious drawback to the Rice-Thomson model is that it treats a fullyformed dislocation on a slip plane intersecting the crack tip, as depicted schematically in Fig. 1(a). The activation energy analysis proceeds by considering a given set of applied loads and then finding stable and unstable configurations which satisfy equations of equilibrium, in terms of $r$ (the radius of the pre-existing loop). As pointed out by ARGON (1987), and more recently Schöck (1991), a dislocation is likely to emerge from a crack tip as a Peierls-like shear distribution of atoms across the slip plane, and hence the Burgers shear displacement of the loop, as well as the radius of the loop, should be considered as the activation parameters. A simple argument given by Argon shows that the consequences for the activation energy should be appreciable: the primary scaling factor for the energy analysis (as will hecome apparent later in this work) is $\mu b^{3}$ ( $\mu$ is the shear modulus and $b$ is the magnitude of the Burgers vector); hence, if the incipient dislocation configuration involves slip of less than a full atomic spacing, then the activation energy should be strongly reduced duc to the power of 3 [the same argument applies as well to the HAASEN (1983) analysis of partial dislocations in silicon]. SCHÖCK (1991) has treated the nucleation from a crack tip within the Peierls framework in an approximate fashion for a straight dislocation and a dislocation loop has been similarly treated by Schöck and Püschl (1991). The activation energy analysis in this paper follows procedures within the Peierls framework set out by RiCE (1992), and further developed by BELTZ and RiCE (1991, 1992), Rice et al. (1992), Bfeltz (1992), Sun et al. (1993), and Sun (1993), in which exact $2 \mathrm{D}$ solutions for incipient shear distributions at a crack tip are determined. 


\section{The Peierls-Nabarro Framework for Nucleation}

To review the Peierls, or Peierls-Nabarro, model for dislocation nucleation, suppose that one of the possible slip planes in a crystal intersects a crack tip. Here we assume that the material is an isotropic elastic solid, that the emergent dislocation is of edge character relative to the crack tip, and that there is negligible effect of tensile stress on the shear response along slip planes (analyses that remove all these simplifications are given in the works cited above). If the solid is loaded, an incipient dislocation may begin to form on a slip plane [see Fig. 1(b)]; under increasing load the dislocation may be emitted if the load reaches a critical value. Alternatively, cleavage decohesion could occur, causing the crack to propagate; this scenario is not addressed in this paper but it is important when evaluating the ductile vs brittle behavior of materials. The shear stress $\tau$ on the potentially active slip plane is taken to be a periodic function of $\delta$ ( $\equiv \delta_{\mathrm{r}}=\delta_{\mathrm{x}}$ for the mode II case to be discussed), the shear displacement discontinuity across the plane. Once $\tau=\tau(\delta)$ is known, the shear displacement profiles $\delta=\delta(x)$ ( $x$ is the distance from the crack tip) as a function of the applied stress intensity factors can be determined via the solution of an integral equation to be introduced below. In the simplest case, when the slip plane is taken to be coplanar with the crack plane, RICE (1992) showed that an incipient edge dislocation becomes unstable when $G$, the "applied" Griffith energy release rate associated with the mode II component of loading, given by $G=(1-v)\left(K_{\mathrm{II}}\right)^{2} / 2 \mu$, attains the value of the unstable stacking energy, corresponding to the first maximum undergone by the energy per unit area of the slip plane, $\Phi=\Phi(\delta)=\int \tau(\delta) \mathrm{d} \delta$. Emission criteria for the case when the slip plane is inclined at some angle $\theta$ may be extracted from the same type of integral equation solution, assuming that a suitable function $\tau(\delta)$ is known, chosen to at least approximately include the effects of tension normal to the slip plane (RICE et al., 1992; BELTZ and RiCE, 1992; Sun et al., 1993).

The shear stress $\tau$ on a slip plane is typically expressed as a function of $\Delta$, the relative atomic displacement undergone by atoms on opposite sides of the cut, which differs from $\delta$ due to an elastic shear strain $\tau / \mu$ acting over a distance $h$ normal to the cut, where $h$ is the interplanar spacing. The simplest modeling assumes that the function $\tau(\delta)$ is given parametrically by the relations (RICE, 1992)

$$
\tau=\left(\pi \gamma_{\mathrm{us}} / b\right) \sin (2 \pi \Delta / b)
$$

with

$$
\delta=\Delta-(b / 2 \pi) \sin (2 \pi \Delta / b) .
$$

An extensive literature exists [see RICE et al. (1992), SUN et al. (1993), and references therein] concerning more realistic forms for $\tau$ vs $\delta$, most of which include a coupling effect due to tensile stresses normal to the slip plane. Equation (2) is commonly associated with the Frenkel model, in which the initial slope of $\tau$ vs $\Delta$ is identified with the shear modulus, giving

$$
\gamma_{\mathrm{us}}=\mu b^{2} / 2 \pi^{2} h
$$

thus within the model $\gamma_{\text {us }}$ is fully determined by $\mu, b$, and $h$.

Results from atomic calculations (RICE et al., 1992; SUN et al., 1993) suggest that 
(4) overestimates the unstable stacking energy for a wide range of materials, or underestimates it, in the case of $\mathrm{Si}$. In this analysis it is convenient to note that until now the parameter $h$ appears only in (4); hence it may be regarded not as the true interplanar spacing but rather as a fitting parameter which allows $\mu$ and $\gamma_{\text {us }}$ to be independently specified for a given material. To be more specific, if $h$ is literally interpreted as the interplanar spacing, $h / b=\sqrt{2}$ for a Shockley partial in the foc lattice, $\sqrt{2 / 4}$ for a Shockley "glide" partial in the diamond cubic lattice, and $\sqrt{2 / 3}$ for a full dislocation ( $\langle 111\rangle$ type slip direction) in the bec lattice, then the Frenkel model rigorously states that, for the three lattices, $\gamma_{u s}$ should be $0.036 \mu b, 0.143 \mu b$, and $0.062 \mu b$, respectively. If, however, it is known that $\gamma_{u s}$ differs from the Frenkel prediction, for example in the glide planes of Si, where the atomic calculation referenced earlier gives $\gamma_{u s}^{(\mathrm{r})}=0.142 \mu b$ an "effective" value of $h / h$, determined from (4), would then be $1 /(0.142) 2 \pi^{2} \approx 0.357$ (vs the actual value of 0.354 ). It is interesting to note that the Frenkel theory overestimates $\gamma_{\text {us }}^{(\mathrm{r})}$ in Si by only about $1 \%$.

The energy per unit area quantities $\Psi(\Delta)$ and $\Phi(\delta)$ are defined such that $\tau \mathrm{d} \Delta=\mathrm{d} \Psi$ and $\tau \mathrm{d} \delta=\mathrm{d} \Phi$. Combining with (3) reveals that $\Phi(\delta)=\Psi(\Delta)-h \tau^{2}(\Delta) / 2 \mu$, and integrating the same equation gives

$$
\begin{aligned}
& \Psi=\gamma_{u s} \sin ^{2}(\pi \Delta / b), \\
& \Phi=\gamma_{u s} \sin ^{4}(\pi \Delta / b) .
\end{aligned}
$$

The latter is expressed in terms of $\delta$ by (3). Figure 2 shows plots of $\Phi(\delta)$ as well as $\tau(\delta)$. Assuming that the slip plane is coplanar with the crack plane and there is mode II loading, RICE (1992) showed that the slip at the crack tip $\delta_{\text {tip }}[=\delta(x)$ at $x=0]$ is given by

$$
G=\Phi\left(\delta_{\mathrm{tip}}\right)=\int_{0}^{\delta_{\mathrm{tip}}} \tau \mathrm{d} \delta,
$$

in any solution which renders stationary the energy functional $U$, the total potential energy of a slipped configuration per unit distance along the crack front. When $\delta_{\text {tip }}$ lies along the branch of the curve labeled $A$ in Fig. 2, the instability state is one of locally minimum energy and corresponds to a stable configuration of an incipient edge dislocation. When $G$ reaches $\gamma_{u s}$, an instability occurs, and the dislocation is emitted. Note that for a given $G$ less than $G_{\text {crit }}\left(G_{\text {crit }}=\gamma_{\text {us }}\right.$ in this simplified case of coincident crack and slip plane under mode Il loading), additional solutions exist. Points $A^{\prime}, A^{\prime \prime}$, etc. correspond to stable incipient dislocations after one, two, etc. dislocations have been emitted from the crack tip. When $\delta_{\text {tip }}$ lies along the portion labeled $C$, the stationary state represents a saddle-point configuration - the slip distribution corresponding to $C$ is unstable due to the fact that the equilibrating load decreases as the distribution expands outward. For a given applied energy release rate $G<G_{\text {crit }}\left(=\gamma_{\text {us }}\right)$ the activation energy is defined as

$$
\Delta U_{\mathrm{atcl}}-U\left[\delta_{\mathrm{sad}}(x)\right]-U\left[\delta_{\min }(x)\right]
$$

where $\delta_{\text {sad }}(x)$ denotes the solution for $\delta(x)$ having $\delta(0)\left(=\delta_{\text {tip }}\right)$ on branch $C$ and $\delta_{\min }(x)$ is the solution on branch $A$. As mentioned earlier, the saddle configuration is 


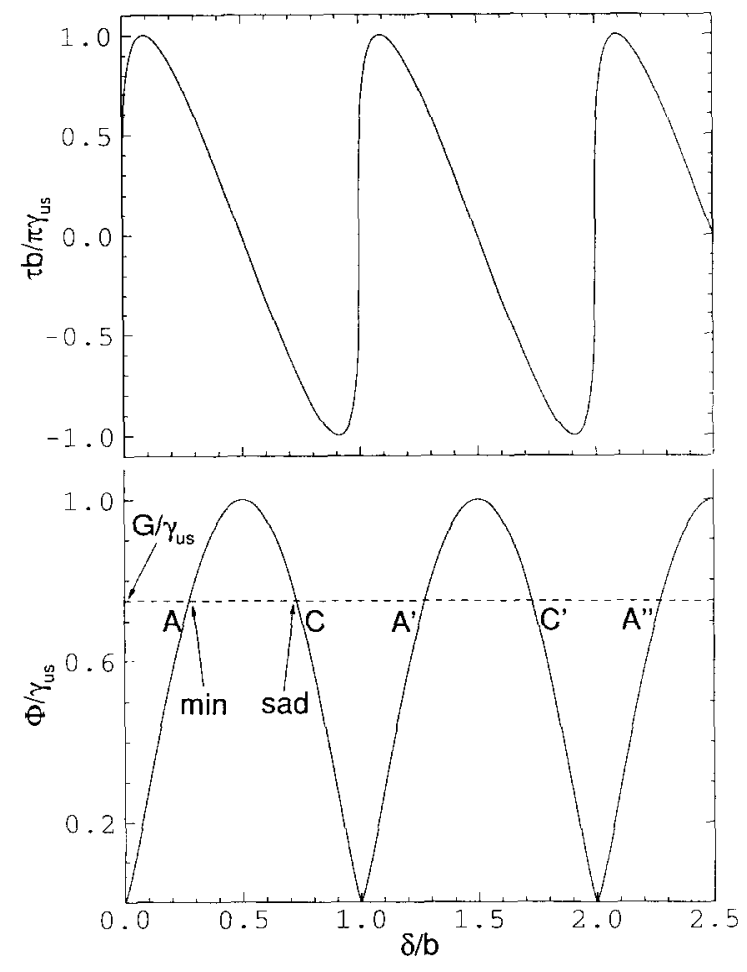

FIG. 2. Shear stress $\tau$ and the slip plane potential $\Phi$ vs displacement discontinuity $\delta$ on the slip plane. Points $A$ and $C$ correspond to values of $\delta$ at the crack tip in the stable (minimum energy) and saddle-point solutions, respectively, at a given load level. The intensity of the mode II loading is characterized by the crack tip energy release rate $G$.

first analyzed by constraining the field to be $2 \mathrm{D}$, so that $\Delta U_{\text {act }}$ is an energy per unit length.

\section{Direct Calculation of the 2D Activation Energy per Unit Length $\Delta U_{\text {act }}$}

The functional $U$ giving energy of a $2 \mathrm{D}$ field per unit length along a crack front may be written for a linear elastic solid under a mode II load as (RICE, 1992)

$$
U[\delta(x)]=U_{0}+\int_{0}^{x} \Phi[\delta(x)] \mathrm{d} x+\frac{1}{2} \int_{0}^{\infty} s[\delta(x)] \delta(x) \mathrm{d} x-\int_{0}^{\infty} \frac{K_{\mathrm{II}}}{\sqrt{2 \pi x}} \delta(x) \mathrm{d} x,
$$

with

$$
s[\delta(x)]=\frac{\mu}{2 \pi(1-v)} \int_{0}^{\infty} \int \frac{\xi}{x} \frac{\mathrm{d} \delta(\xi) / \mathrm{d} \xi}{x-\xi} \mathrm{d} \xi .
$$

The first term in (8), $U_{0}$, denotes the energy of the unslipped solid, i.e. the energy of 
the loaded solid when $\delta(x)$ is constrained to zero. The second term is the energy gained on the slip plane when $\delta(x)$ develops, and the remainder of the terms account for the energy change of the material outside the slip plane and of the loading system due to the introduction of $\delta(x)$. The functional $s[\delta(x)]$ gives the amount by which the shear stress $\tau$ decreases upon introduction of the slip, with its "kernel" function $[\mu / 2 \pi(1-v)] \sqrt{\xi / x} /(x-\xi)$ identified as the shear stress at distance $x$ due to a unit edge dislocation located at position $\xi$ [see, e.g., THOMSON (1986) or BFLTZ (1992)]. Rendering (8) stationary with respect to $\delta(x)$ yields the integral equation

$$
\tau[\delta(x)] \equiv \Phi^{\prime}[\delta(x)]=\frac{K_{11}}{\sqrt{2 \pi x}}-s[\delta(x)]
$$

which corresponds to enforcing stress equilibrium along the slip plane. Stable, or minimum energy, solutions $\dot{\delta}_{\min }(x)$ of $(10)$ have been determined numerically by BELTZ and RiCE (1991, 1992) and BeLTZ (1992) in connection with the dislocation emission problem (i.e., in the range where $G$ and $\delta_{1 i p}$ lie on branch $A$ of the energy curve in Fig. 2). Solutions are shown as solid lines for $\gamma_{u s}=\mu b / 2 \pi^{2}$ and $v=0.3$ in Fig. 3(a) at various load levels up to instability. In order to determine the saddle-point solutions, a second solution of $(10)$ must be determined for each level of applied load. These solutions are found via the method used to determine the stable solutions, except that initial guesses of $\delta(x)$ are used that are greater than the solution corresponding to $G=G_{\text {cril }}$, and which have a $\dot{\delta}_{\text {tip }}$ given by the larger solution of (6). These solutions are shown as dashed lines in Fig. 3(a) for the same values of $G$ as were used to determine the stable solutions, shown as solid lines. For more general conditions than assumed here (e.g. slip plane inclined with respect to crack plane, screw and edge components of emergent dislocation, tension-shear coupling, and mixed-mode loading, all as dealt with in references cited above) $G_{\text {crit }}$ should be interpreted as the maximum $G$ for which a stable solution can be determined. Thus, while $G_{\text {crit }}=\gamma_{u \text { u }}$ in the specific case analyzed here, we shall generally phrase results in terms of the ratio $G / G_{\text {crit }}$, supposing them to be at least approximately valid in those more general situations.

Inserting (8) into (7) and simplifying with the help of (10) gives the following expression for the 2D activation energy, which can be simply evaluated numerically for a given pair $\delta_{\min }(x)$. and $\delta_{\text {sid }}(x)$ :

$$
\Delta U_{\mathrm{ac} 1}^{2 \mathrm{D}}=\int_{0}^{x}\left\{\hat{\Phi}\left[\delta_{\mathrm{sad}}(x)\right]-\hat{\Phi}\left[\delta_{\min }(x)\right]\right\} \mathrm{d} x-{ }_{2}^{1} K_{\mathrm{Il}} \int_{0}^{x} \frac{\delta_{\mathrm{sad}}(x)-\delta_{\mathrm{min}}(x)}{\sqrt{2 \pi x}} \mathrm{~d} x
$$

where $\hat{\Phi}(\delta) \equiv \Phi(\delta)-\delta \Phi^{\prime}(\delta) / 2$. Evaluation of (11) is carried out for $\gamma_{\mathrm{us}}=\mu b / 2 \pi^{2}$ and $v=0.3$. As will be discussed later, the calculation is extremely insensitive to these values, however. In Fig. 4 , the results are plotted as a function of applied load as a solid line. Table 1 shows the results for specific $G$ values. The first column gives $\Delta U_{\text {aci }}^{2 \mathrm{D}}$ (an energy per unit dislocation length). The entry in each of the remaining columns gives an estimate of the $3 \mathrm{D}$ activation energy $\Delta E$ obtained by writing $\Delta E=7.5 b \Delta U_{\text {act }}^{2 \text { D }}$ (i.e. assuming an activated dislocation length of $7.5 \mathrm{~b}$ ), for a Shockley partial dislocation in $\mathrm{Cu}$, a full dislocation in $\mathrm{Fe}$, and a partial dislocation in $\mathrm{Si}$. The 
(a)

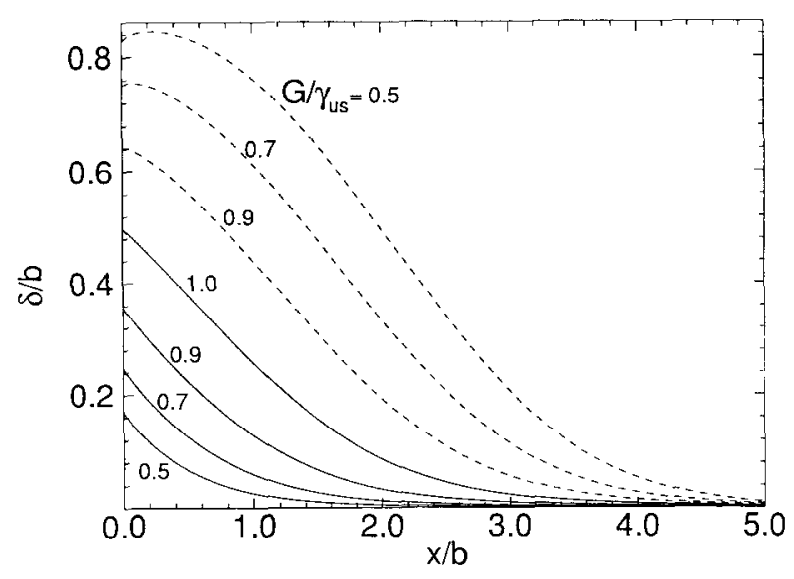

(b)

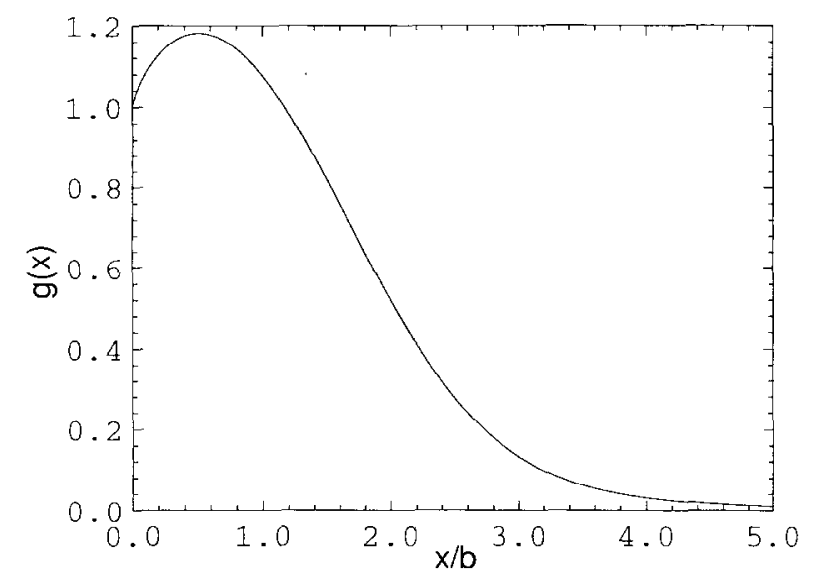

FIG. 3. (a) Displacement profiles for various loads; the solid lines are the minimum energy solutions and the dashed lines are the corresponding saddle-point solutions. (b) Eigenfunction $g(x)\left[=\partial \delta\left(x ; \delta_{\text {tip }}\right) / \partial \delta_{\text {tip }}\right.$, evaluated at $\delta_{\text {tip }}=b / 2$ ] determined as part of the $2 \mathrm{D}$ asymptotic analysis. All plots are for $\gamma_{\mathrm{us}}=\mu b / 2 \pi^{2}$ and $v=0.3$.

$\Delta E$ estimated are listed in units of $k T$ as evaluated at room temperature; implications for thermally activated nucleation are discussed later in this paper.

\section{Asymptotic Calculation of the 2D Activation Energy}

In order to more simply evaluate the $2 \mathrm{D}$ activation energy near critical loading, and to provide a basis for the 3D analysis to come, we present here an asymptotic calculation of the activation energy per unit length, good for small deviations of the applied load from the critical load for emission. The method proceeds via a perturbation analysis of the shear distribution. It is useful to regard a given shear profile, satisfying $(10)$, as being a function of $x$ and of $\delta_{\text {tip }}$, i.e. $\delta=\delta\left(x ; \delta_{\text {tip }}\right)$. Note that a pair of $\delta_{\text {tip }}$ values corresponds to a given $G$ (less than $G_{\text {crii }}$ ) by (6) and Fig. 2, so one 


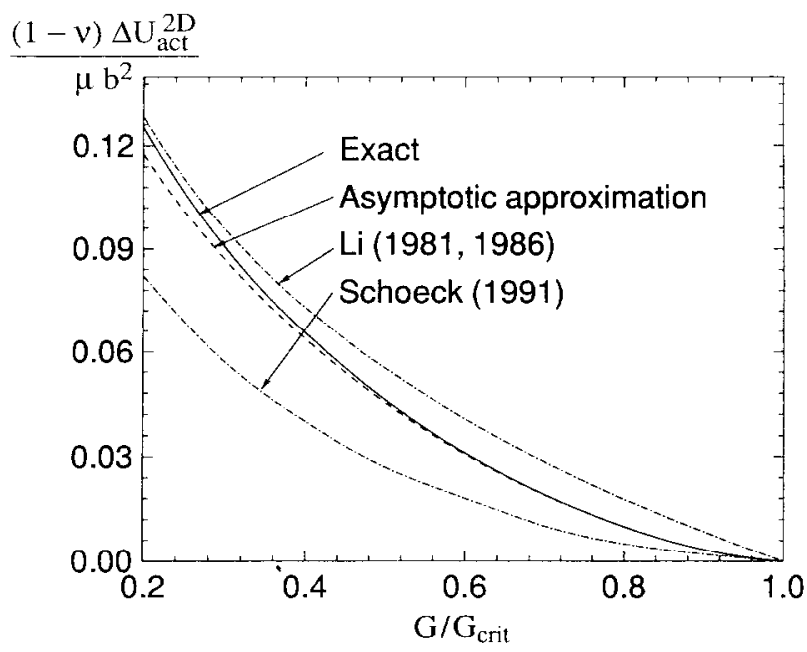

Fig. 4. Activation energy $\Delta U$ (2D) per unit length vs loading. The solid line is the exact result from numerical solutions of the Peierls model. The dashed line is the result of the asymptotic expansion about the critical loading. The dashed dotted lines show the predictions of LI $(1981,1986)$ based on the Rice

Thomson model, and Sснӧск (1991) based on an approximate implementation of the Peicrls model.

$\delta\left(x ; \delta_{\mathrm{tin}}\right)$ is $\delta_{\min }(x)$ and the other is $\delta_{\text {sad }}(x)$. Suppose $\delta=f(x)$ is the shear profile at instability $\left(G=G_{\text {crit }}\right)$; in the case of a mode II shear crack with the Frenkel form, $f(x)=\delta(x ; b / 2)$, which corresponds to the last solid line in Fig. 3(a). If the applied energy release rate $G$ is slightly less than $G_{\text {crii }}$, the shear distribution may be written as

$$
\delta(x)=f(x)+g g(x),
$$

where $\varepsilon=\delta_{\text {ip }}-b / 2$ characterizes the extent of the perturbation and $g(x)$ is defined by

TABle 1. Activation energies: $\mathrm{Cu}, \mathrm{Fe}$, and $\mathrm{Si} 3 D$ estimates based on (exact) $2 D$ calculation $\dagger$

\begin{tabular}{ccccc}
\hline$\frac{G}{G_{\text {crit }}}$ & $\frac{(1-v) \Delta U_{\mathrm{act}}^{2 \mathrm{D}}}{\mu b^{2}}$ & $\frac{\Delta E_{\mathrm{Cu} \text {, partial }}}{k T_{\text {room }}}$ & $\frac{\Delta E_{\mathrm{Fe}, \text { fuld disl. }}}{k T_{\text {room }}}$ & $\Delta E_{\text {Si. partial }}$ \\
\cline { 2 - 3 } 0.1 & $1.67 \times 10^{-1}$ & 59.0 & 454.1 & $k T_{\text {room }}$ \\
0.2 & $1.25 \times 10^{-2}$ & 44.3 & 340.7 & 256.2 \\
0.3 & $9.05 \times 10^{-2}$ & 32.0 & 246.6 & 192.2 \\
0.4 & $6.55 \times 10^{-2}$ & 23.1 & 178.5 & 139.1 \\
0.5 & $4.62 \times 10^{-2}$ & 16.4 & 125.9 & 100.7 \\
0.6 & $3.10 \times 10^{-2}$ & 11.0 & 84.5 & 71.0 \\
0.7 & $1.90 \times 10^{-2}$ & 6.72 & 51.8 & 47.7 \\
0.8 & $9.85 \times 10^{-3}$ & 3.48 & 26.9 & 29.3 \\
0.9 & $3.32 \times 10^{-3}$ & 1.17 & 9.05 & 15.2 \\
1.0 & 0 & 0 & 0 & 5.10 \\
\hline
\end{tabular}

$+\Delta U_{\mathrm{ac}}^{2 \mathrm{D}}$ is the activation energy per unit length for $2 \mathrm{D}$ configurations. The entries in the remaining columns are the $2 \mathrm{D}$ results multiplied by $7.5 \mathrm{~b}$ and the appropriate material constants. 


$$
g(x) \equiv\left[\frac{\partial \delta\left(x ; \delta_{\text {tip }}\right)}{\partial \delta_{\text {tip }}}\right]_{\delta_{\text {tip }}-b / 2}
$$

Note that $\varepsilon$ has units of length and $g(x)$ is dimensionless. A relation satisfied by the function $g(x)$ may be determined by differentiating (10) with respect to $\delta_{\text {uip }}$ and evaluating for $\delta(x)=f(x)$, noting that the term $\partial K_{\mathrm{II}} / \partial \delta_{\text {tip }}$ vanishes because $G$, and hence $K_{\mathrm{Il}}$, undergoes a maximum at the instability point characterized by $f(x)$. The result shows that $g(x)$ satisfies the eigenproblem

$$
\Phi^{\prime \prime}[f(x)] g(x)=-s[g(x)] .
$$

An implication is that the function of $x$ generated by $s[g(x)]$ is finite at $x=0$; i.e. considered as a slip distribution, $g(x)$ would induce no singularity at the crack tip. The function $g(x)$, for $\gamma_{u s}=\mu b / 2 \pi^{2}$ and $v=0.3$, is plotted in Fig. 3 (b).

The algebraic manipulations to follow rely on the following relation, which follows from the elastic reciprocal theorem :

$$
\int_{0}^{x} s\left[\delta_{1}(x)\right] \delta_{2}(x) \mathrm{d} x=\int_{0}^{x_{1}} s\left[\delta_{2}(x)\right] \delta_{1}(x) \mathrm{d} x .
$$

Equation (15) holds for any pair of shear distributions $\delta_{1}(x)$ and $\delta_{2}(x)$; they need not satisfy (10). Now the total energy $U$ of the system may be written, using the point of instability as a reference, as

$$
\begin{aligned}
& U=U(\varepsilon)=U_{\text {crit }}+\int_{0}^{x}\{\Phi[f(x)+\varepsilon g(x)]-\Phi[f(x)]\} \mathrm{d} x \\
& +\frac{1}{2} \int_{0}^{\infty}\{s[f(x)+\varepsilon g(x)][f(x)+\varepsilon g(x)]-s[f(x)] f(x)\} \mathrm{d} x-\int_{0}^{\infty} \frac{K_{\mathrm{II}}}{\sqrt{2 \pi x}} \varepsilon g(x) \mathrm{d} x .
\end{aligned}
$$

Equation (10) when evaluated at the critical state shows that

$$
\tau[f(x)]=\Phi^{\prime}[f(x)]=\frac{K_{11}^{\text {crit }}}{\sqrt{2 \pi x}}=-s[f(x)] .
$$

With the help of the reciprocal theorem and (14) and (17), the energy may then be written as

$$
U=U_{\mathrm{crit}}-Q(\varepsilon)+\left(K_{\mathrm{II}}^{\mathrm{crit}}-K_{\mathrm{II}}\right) \varepsilon \beta
$$

where

$$
Q(\varepsilon)=-\int_{0}^{x}\left\{\Phi[f(x)+\varepsilon g(x)]-\Phi[f(x)]-\varepsilon g(x) \Phi^{\prime}[f(x)]-\frac{1}{2} \varepsilon^{2} g^{2}(x) \Phi^{\prime \prime}[f(x)]\right\} \mathrm{d} x,
$$

$$
\beta \equiv \int_{0}^{\infty} \frac{g(x)}{\sqrt{2 \pi x}} \mathrm{~d} x \approx 1.255 \sqrt{b}
$$


with the numerical value given for $\gamma_{u s}=\mu b / 2 \pi^{2}$. Now consider the function $Q(\varepsilon)$ and its derivatives with respect to $s$ evaluated at $c=0$. One sees that

$$
Q(0)=Q^{\prime}(0)=Q^{\prime \prime}(0)=0,
$$

and that

$$
Q^{\prime \prime \prime}(0)=-\int_{0}^{\infty} g^{3}(x) \Phi^{\prime \prime \prime}[f(x)] \mathrm{d} x,
$$

where $\Phi^{\prime \prime \prime}(\delta) \equiv \mathrm{d}^{3} \Phi(\delta) / \mathrm{d} \delta^{3}$. Hence the Taylor series for $Q$, expanded to third order, trivially reduces to

$$
Q(\varepsilon)=\frac{1}{3 !} Q^{\prime \prime \prime}(0) \varepsilon^{2}
$$

and the energy may now be written as

$$
U=U_{\text {crit }}-\frac{1}{6} Q^{\prime \prime \prime}(0) \varepsilon^{3}+\left(K_{11}^{\mathrm{crit}}-K_{\mathrm{II}}\right) \varepsilon \beta
$$

The stationary points now may be determined by ensuring that the derivatives with respect to $\varepsilon$ vanish, which requires that

$$
-{ }_{2}^{1} Q^{\prime \prime \prime}(0) \varepsilon^{2}+\left(K_{11}^{\mathrm{cril}}-K_{1 \mathrm{I}}\right) \beta=0 .
$$

Since $Q^{\prime \prime \prime}(0)>0$, this equation has solutions for $\varepsilon$ only when $K_{\mathrm{II}}<K_{\mathrm{II}}^{\mathrm{crit}}$, as expected. and solving for $\varepsilon$ yields two solutions

$$
\varepsilon= \pm \sqrt{\frac{2 \beta\left(K_{11}^{\mathrm{cri}}-K_{\mathrm{II}}\right)}{Q^{\prime \prime \prime}(0)}}
$$

which may be alternatively written in terms of $G$ as

$$
\varepsilon= \pm h^{(2 \beta / \sqrt{b})^{1 / 2}\left[2 \mu b /(1-v) \gamma_{\text {us }}\right]^{1: 4}}\left(1 \quad \sqrt{G / G_{\text {crt }}}\right)^{1: 2} \approx \pm 0.4543 b\left(1-\sqrt{G / G_{\text {crit }}}\right)^{1 / 2}
$$

where the numerical factor in the latter form, also verified numerically, can be obtained from equating $G$ to $\Phi$ of (6) and (5b), and then expanding about $G=\gamma_{\text {us }}$ and $\delta=b / 2$. Writing the asymptotic solution as $\delta(x)=f(x)+\varepsilon g(x)$, the stable minimum corresponds to the negative root and the saddle point corresponds to the positive root. Finally, (7) and (23) may be invoked to determine the activation energy per unit length

$$
\Delta U_{\mathrm{illt}}^{2 \mathrm{D}}=\frac{2}{3} \frac{\left[2 \beta\left(K_{\mathrm{II}}^{\mathrm{crit}}-K_{\mathrm{II}}\right)\right]^{3 ; 2}}{\left[Q^{\prime \prime \prime}(0)\right]^{1 ; 2}}
$$

In terms of the applied energy release rate, (27) can be rewritten as 


$$
\Delta U_{\text {act }}^{2 \mathrm{D}}=\frac{2^{13 / 4}}{3}\left(\frac{\mu G_{\text {crit }}}{1-v}\right)^{3 / 4} \frac{\left[\beta\left(1-\sqrt{G / G_{\text {crit }}}\right)\right]^{3 / 2}}{\left[Q^{\prime \prime \prime}(0)\right]^{1 / 2}}
$$

or in dimensionless terms as

$$
\frac{(1-v) \Delta U_{\mathrm{act}}^{2 \mathrm{D}}}{\mu b^{2}}=m\left(\gamma_{\mathrm{us}} / \mu b, v\right)\left(1-\sqrt{G / G_{\mathrm{crit}}}\right)^{3 / 2}
$$

where the dimensionless factor $m=m\left(\gamma_{\mathrm{us}} / \mu b, v\right)$ is given by

$$
m=\frac{8(\beta / \sqrt{b})^{3 / 2}\left[2(1-v) \gamma_{\text {us }} / \mu b\right]^{1 / 4}}{3} \frac{\left[b^{2} Q^{\prime \prime \prime}(0) / \gamma_{\text {us }}\right]^{1 / 2}}{}
$$

where all the terms involving $\beta$ and $Q^{\prime \prime \prime}(0)$ are arranged as dimensionless combinations which depend on $\gamma_{u s} / \mu b$ and $v$.

The results, expressed as $\Delta U_{\text {act }}^{2 \mathrm{D}}$ vs applied $G$, are plotted in Fig. 4 for $\gamma_{\text {us }}=\mu b / 2 \pi^{2}$ and $v=0.3$. The solid line gives the activation energy as calculated exactly in the previous section, and the dashed line gives the asymptotic result, which is seen to be remarkably accurate over the entire range $G / G_{\text {crit }}>0.2$ shown. The dimensionless function $m$ has values of 0.2869 for $\mu b / 2 \pi^{2} \gamma_{\text {us }}=\sqrt{2} / 4,0.2865$ for $\mu b / 2 \pi^{2} \gamma_{\text {us }}=\sqrt{2 / 3}$, 0.2864 for $\mu b / 2 \pi^{2} \gamma_{\text {us }}=1.0$, and 0.2863 for $\mu b / 2 \pi^{2} \gamma_{\text {us }}=\sqrt{2}$ (all for $v=0.3$ ), indicating an extremely weak dependence on $\gamma_{\mathrm{us}} / \mu b$. Thus $(1-v) \Delta U_{\mathrm{ac}}^{2 \mathrm{D}} / \mu b^{2}$ is, essentially, dependent only on $G / G_{\text {eril }}$; the ratio $\gamma_{u s} / \mu b$ is still important in determining the activation energy, in as far as it affects the calculation of $G_{\text {crit }}\left(=\gamma_{\text {us }}\right.$ for the present case of coincident crack and slip planes).

\section{D Asymptotic Calculation of the Saddle Point loop Shape nNd ACTIVATION ENERGY}

In the $3 \mathrm{D}$ analysis, it is assumed that the saddle-point configuration involves a $3 \mathrm{D}$ elastic field associated with a localized outward protrusion of slip from the stable 2D incipient dislocation distribution corresponding to branch $A$ of Fig. 2. Here, the reference state is the $2 \mathrm{D}$ pre-nucleation state in the above analysis, i.e. the state given by the negative root of $\varepsilon$ in (25). It is convenient, however, to think of $\varepsilon$ as a positive number for the pre-nucleation state, so the shear distribution is written as $\delta(x)=f(x)-\varepsilon g(x)$, with $\varepsilon$ interpreted as $b / 2-\delta_{\text {tip }}$. The saddle-point distribution, which now is taken to be $3 \mathrm{D}$, is assumed to be approximately expressible as $\delta(x, z)=f(x)-\varepsilon g(x)+a(z) g(x)$, where $a(z)$ is a "shape" function characterizing the extent of the outward perturbation, which we determine so as to render the energy stationary. The coordinate $z$ lies along the crack tip and $z=0$ is taken to be the center of the perturbation (see Fig. 5). Note that the trivial solution $a(z)=0$ corresponds to the pre-nucleation state and $a(z)=2 \varepsilon$ corresponds to the $2 \mathrm{D}$ saddle-point distribution discussed in the previous section.

The functional $s[\delta(x, z)]$, relating the stress on the slip plane (coplanar with the crack) to the displacement distribution, is considerably more complicated than its $2 \mathrm{D}$ counterpart. However, it has been given by $\mathrm{G} \Lambda O$ (1988) and GAO and RICE (1989), 


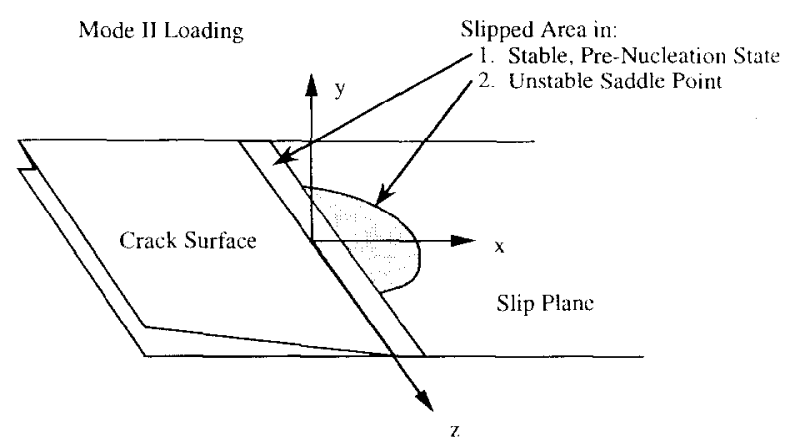

Fig. 5. Three-dimensional geometry and coordinate system.

with additional simplification by H. GAO (private communication, 1991); see the Appendix. Whenever the argument of $s$ does not depend on $z$, however, it may be interpreted as its $2 \mathrm{D}$ representation given in (9). The $3 \mathrm{D}$ analog to the reciprocal theorem given by $(15)$ is

$$
\int_{x}^{\infty} \int_{0}^{\infty} s\left[\delta_{1}(x, z)\right] \delta_{2}(x, z) \mathrm{d} x \mathrm{~d} z=\int_{x}^{x} \int_{0}^{\infty} s\left[\delta_{2}(x, z)\right] \delta_{1}(x, z) \mathrm{d} x \mathrm{~d} z .
$$

The functional characterizing the difference in energy between the $2 \mathrm{D}$ pre-nucleation state and the saddle-point distribution is

$$
\begin{aligned}
\Delta E=\int_{x}^{x} & \int_{0}^{x}\{\Phi[f(x)-\varepsilon g(x)+a(z) g(x)]-\Phi[f(x)-\varepsilon g(x)]\} \mathrm{d} x \mathrm{~d} z \\
+ & \frac{1}{2} \int_{x}^{x} \int_{01}^{x}\{s[f(x)-\varepsilon g(x)+a(z) g(x)][f(x)-\varepsilon g(x)+a(z) g(x)] \\
& s[f(x) \quad g(x)][f(x)-\varepsilon g(x)]\} \mathrm{d} x \mathrm{~d} z-K_{\mathrm{II}} \int_{x}^{x} \int_{0}^{x} \frac{a(z) g(x)}{\sqrt{2 \pi x}} \mathrm{~d} x \mathrm{~d} z .
\end{aligned}
$$

Proceeding in an analogous manner as with the 2D energy functional, the $3 \mathrm{D}$ energy functional simplifies to

$$
\begin{aligned}
& \Delta E=\int_{x}^{\infty} \int_{0}^{\infty}\{\Phi[f(x)-g g(x)+a(z) g(x)]-\Phi[f(x)-g g(x)] \\
& \left.-a(z) g(x)\left(\Phi^{\prime}[f(x)]-\varepsilon g(x) \Phi^{\prime \prime}[f(x)]\right)-\frac{1}{2} a^{2}(z) g^{2}(x) \Phi^{\prime \prime}[f(x)]\right\} \mathrm{d} x \mathrm{~d} z \\
& +\frac{1}{2} \int_{0}^{x} \int_{0}^{x}\{s[a(z) g(x)]-a(z) s[g(x)]\} a(z) g(x) \mathrm{d} x \mathrm{~d} z \\
& +\left(K_{\mathrm{II}}^{\mathrm{crit}}-K_{\mathrm{II}}\right) \int_{x}^{x} \frac{\int_{0}^{x}}{\sqrt{2 \pi x}} \mathrm{~d} x \mathrm{~d} z
\end{aligned}
$$


To simplify further, it is assumed that $\Phi$ may be expanded as a Taylor series about $f(x)$ [valid of course for small $\varepsilon$ and $a(z)$, and assuming the derivatives of $\Phi(\delta)$ with respect to $\delta$ exist], just as was done in the $2 \mathrm{D}$ analysis above, with the result that

$$
\begin{aligned}
& \Phi[f(x)-\varepsilon g(x)+a(z) g(x)]=\Phi[f(x)]+\Phi^{\prime}[f(x)] g(x)[a(z)-\varepsilon] \\
& +\frac{1}{2} \Phi^{\prime \prime}[f(x)] g^{2}(x)[a(z)-\varepsilon]^{2}+\frac{1}{6} \Phi^{\prime \prime \prime}[f(x)] g^{3}(x)[a(z)-\varepsilon]^{3}, \quad \text { (34a) } \\
& \Phi[f(x)-\varepsilon g(x)]=\Phi[f(x)]-\Phi^{\prime}[f(x)] \varepsilon g(x)+\frac{1}{2} \Phi^{\prime \prime}[f(x)] \varepsilon^{2} g^{2}(x)-\frac{1}{6} \Phi^{\prime \prime \prime}[f(x)] \varepsilon^{3} g^{3}(x) .
\end{aligned}
$$

Inserting (34a) and (34b) into (33) and simplifying gives

$$
\begin{aligned}
\Delta E=-\frac{1}{6} Q^{\prime \prime \prime}(0) \int_{-\infty}^{-x}\left\{[a(z)-\varepsilon]^{3}+\varepsilon^{3}\right\} \mathrm{d} z+\left(K_{\mathrm{II}}^{\text {crit }}-K_{\mathrm{II}}\right) \beta \int_{-\infty}^{\infty} a(z) \mathrm{d} z \\
\quad+\frac{1}{2} \int_{\infty}^{\infty} \int_{0}^{\infty}\{s[a(z) g(x)]-a(z) s[g(x)]\} a(z) g(x) \mathrm{d} x \mathrm{~d} z,
\end{aligned}
$$

where $Q^{\prime \prime \prime}(0)$ and $\beta$ are the same as in the $2 \mathrm{D}$ analysis. In order to determine the functions $a(z)$ which make $\Delta E$ stationary, the first variation of $\Delta E$ must vanish for arbitrary variations of $a(z)$. Using (24) to replace the term with the $K_{11}$ s, this gives

$$
0=-\frac{1}{2} Q^{\prime \prime \prime}(0)\left\{[a(z)-\varepsilon]^{2}-\varepsilon^{2}\right\}+\int_{0}^{\infty}\{s[a(z) g(x)]-a(z) s[g(x)]\} g(x) \mathrm{d} x .
$$

Re-arranging gives the following integral equation for $a(z)$, the solutions of which should correspond to extrema of energy:

$$
Q^{\prime \prime \prime}(0)\left[\varepsilon a(z)-\frac{1}{2} a^{2}(z)\right]=\int_{0}^{\infty}\{a(z) s[g(x)]-s[a(z) g(x)]\} g(x) \mathrm{d} x .
$$

Observe that $a(z)=0$ and $a(z)=2 \varepsilon,-\infty<z<+\infty$, are the solutions corresponding to the $2 \mathrm{D}$ minimum energy and saddle-point states. We wish instead to find a solution with non-constant $a(z)$, but with $a(z) \rightarrow 0$ as $|z| \rightarrow \infty$, corresponding to the 3D saddlepoint state. Inserting (37) into (35) yields the simplified expression for the activation energy

$$
\Delta E=\frac{Q^{\prime \prime \prime}(0)}{12} \int_{-\infty}^{\infty} a^{3}(z) \mathrm{d} z .
$$

Because the reference state used in (32) is exactly the pre-nucleation state, the energy in (38) is the activation energy, consistent with the definition in (7). It must be understood, however, that the function $a(z)$ must satisfy (37) before (38) is evaluated.

The numerical procedure for solving (37) proceeds by discretizing the function $a(z)$, i.e., $a_{i}=a\left(z_{i}\right)$, over the integration interval. The integral equation may be then regarded as a nonlinear set of algebraic equations, which may be iterated to consistency via the Newton-Raphson method for a given value of $\varepsilon$. When initial guesses covering a wide range of shapes and sizes (e.g. Gaussian curves of various widths and rec- 


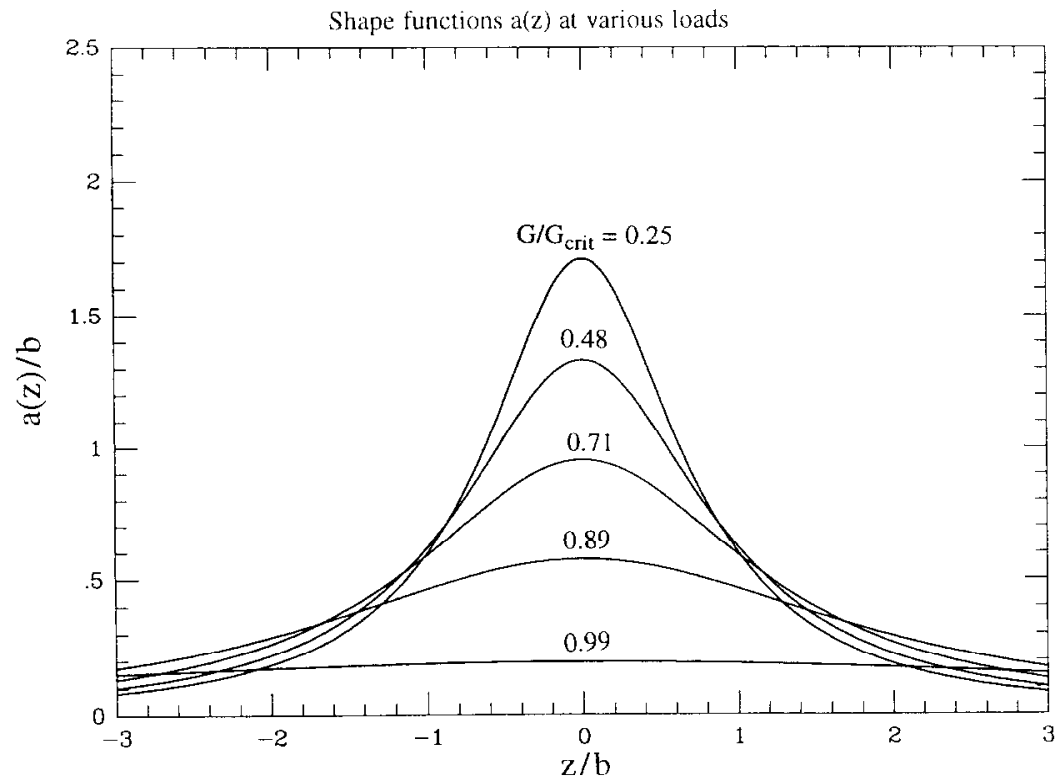

FIG. 6. Shape functions $a(z)$ of 3D asymptotic analysis for various values of $G / G_{\text {cril }}$ (corresponding to $\varepsilon / h=0.45,0.35,0.25,0.15$, and 0.05$)$; the slip at the crack tip is $\delta_{\mathrm{ti} \Gamma}=b / 2-\varepsilon+a(z)$.

tangular boxes with varying degrees of roundness in the corners) are input, the algorithm either: (i) converges to $a(z)=0$, (ii) converges to $a(z)=2 \varepsilon$, (iii) converges to $a(z)$ equal to the solutions shown in Fig. 6 and discussed in the following section, or (iv) diverges. Evaluation of the integral on the right-hand side of (37) is exacerbated by the fact that the $3 \mathrm{D}$ form of the $s$ operator involves the evaluation of slowlyconvergent double-integrals, leading to an unwieldy triple integral overall. The integrands involved in the evaluation of $s$ are functions of $x, \tilde{x}$, and $z-\tilde{z}$ (where $\tilde{x}$ and $\tilde{z}$ are the actual integration variables), thereby making the integral of the second term in (37) a convolution of $a(z)$ with another function. The integral may be evaluated by combining the terms involved in the right-hand side of (37) and integrating first over $x$ and $\tilde{x}$. More details concerning the numerical procedure may be found in the Appendix.

As a confirmation that the solutions shown in Fig. 6 are indeed saddle-point solutions, the energy functional in (35) is regarded as a function of the variables $a_{i}$ characterizing a solution and its Hessian is numerically evaluated. The eigenvalues of the Hessian are then calculated and, in all cases, there are a mixture of negative and positive values. The mixture of eigenvalues implies that the Hessian is indefinite, and hence the calculated solutions must be saddle points.

\section{Numerical Results AND Discussion}

The $2 \mathrm{D}$ results are shown in Fig. 4 ; the exact calculation of the activation energy (per unit length) is shown as a solid line, the asymptotic approximation is shown as 
a dashed line, and the result derived by LI $(1981,1986)$ [see (1)] based on the RiceThomson model is shown as an alternating dashed-dotted line, which overestimates the more exact results here. The SCHÖck (1991) 2D result is also shown, which underestimates the activation energies calculated here by about $40 \%$. The scaling factor used, i.e. $\mu b^{2} /(1-v)$ turns out to be particularly convenient because the numerical results become relatively insensitive to $\gamma_{\text {us }} / \mu b$ when plotted as a function of $G / G_{\text {crit }}$ $\left(=G / \gamma_{u s}\right)$. This is discussed earlier in connection with the $2 \mathrm{D}$ asymptotic solution and it also holds for the exactly calculated results. Of course, the activation energy predicted by (1) is entirely independent of $\gamma_{u s} / \mu b$.

In order to quantify the concept of thermal activation, it is essential to realize that the activation process must involve a finite amount of energy supplied locally to the dislocation line due to atomic vibrations. The process is inherently $3 \mathrm{D}$, so a proper treatment, as attempted in an asymptotic approximation in this paper, would determine the saddle-point configuration, consisting of a local protrusion of a dislocation loop. The activation process could not adequately be modelled in $2 \mathrm{D}$ because an infinite amount of energy would have to be supplied to the dislocation line to achieve the saddle-point configuration. A simple approximation is to multiply the energy per unit length (from the 2D analysis) by a fixed number of atomic spacings, say 5-10, based on the $3 \mathrm{D}$ asymptotic results to be discussed. Table 1 shows the results from the 2D analysis (assuming an activated dislocation length of $7.5 \mathrm{~b}$, i.e. $\Delta E \approx 7.5 b \Delta U_{\mathrm{acl}}^{2 \mathrm{D}}$ ) for partial dislocations in $\mathrm{Cu}$ and $\mathrm{Si}$ and a full dislocation in $\mathrm{Fe}$; this scheme both underestimates and overestimates $\Delta E$ when compared to the $3 \mathrm{D}$ results, depending on the $G / G_{\text {crit }}$ ratio. The $\Delta E$ estimates are listed in units of $\mathrm{kT}$ as evaluated at room temperature. Material constants used in the various calculations are tabulated in Table 2.

The $3 \mathrm{D}$ results are plotted in Fig. 7 as a solid line. For comparison, the $2 \mathrm{D}$ result (the solid line from Fig. 4) is multiplied by $5 b$ and by $10 b$, and plotted as dashed lines in Fig. 7. It is seen that $5 b$ is too small of a factor to use in conjunction with the $2 \mathrm{D}$ results. For larger loadings, a factor of as high as $10 \mathrm{~b}$ is appropriate, and as high as $17 b$ for loadings very near to critical (e.g. $G \approx 0.9 G_{\text {crit }}$ ). Additionally, the $3 \mathrm{D}$ result by SCHÖCK and PüschL (1991) is shown, which varies considerably from the present result. Table 3 also gives specific values of the $3 \mathrm{D}$ result for the materials $\mathrm{Cu}, \alpha-\mathrm{Fe}$ and $\mathrm{Si}$.

The functions $a(z)$, which give the $z$-dependence of the saddle point for the $3 \mathrm{D}$ solutions, are plotted in Fig. 6 for various Ioad levels, corresponding to $\varepsilon / h=0.05$, $0.15,0.25,0.35$, and 0.45 . Note that $a(z)$ resembles a Gaussian "bell" curve and undergoes a maximum value of between $3 \varepsilon$ and $4 \varepsilon$, thus the furthest extent of the 3D protrusion is greater than the $2 \varepsilon$ protrusion predicted by the $2 \mathrm{D}$ analysis. This feature

TABLE 2. Material constants $\left(\mathrm{k}=1.381 \times 10^{-2.3} \mathrm{~J} / \mathrm{K}\right)$

\begin{tabular}{cccc}
\hline Property & Cu (partial) & Fe (full disl.) & Si (partial) \\
\hline$\mu($ GPa $)$ & 40.8 & 69.3 & 60.5 \\
$v$ & 0.324 & 0.291 & 0.218 \\
$b(\mathrm{~m})$ & $1.4757 \times 10^{-10}$ & $2.4825 \times 10^{-10}$ & $2.2172 \times 10^{-10}$ \\
\hline
\end{tabular}




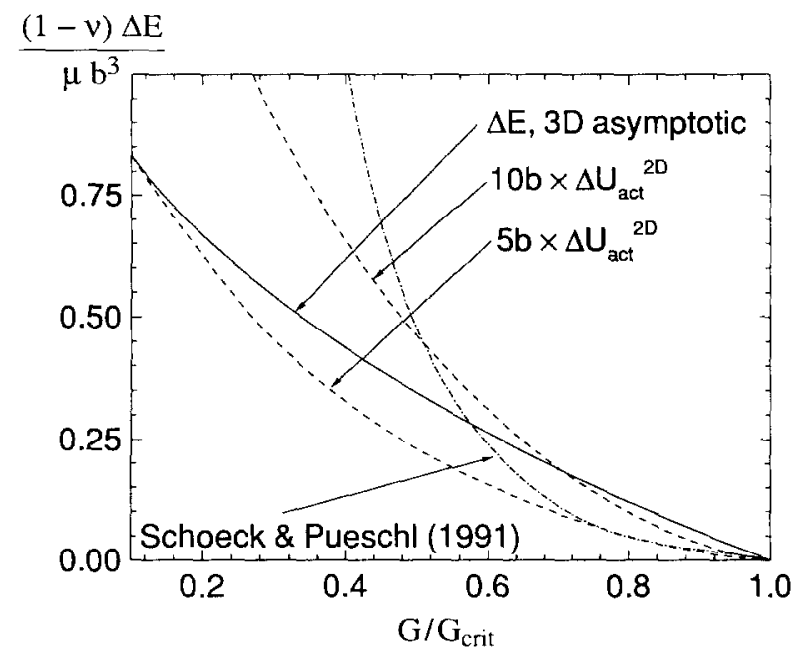

FIG. 7. Activation energy $\Delta E$ (3D) vs loading. The solid line is the asymptotic result and the dashed lines are the $2 \mathrm{D}$ "exact" result multiplied by $5 b$ and by $10 b$; dashed-dotted line is result of SCHÖCK and PüsChL (1991).

may be qualitatively rationalized as follows: one consequence of the $3 \mathrm{D}$ nature of the problem is that variations in the function $a(z)$ give rise to a "screw"-like contribution to $s[a(z) g(x)]$, that is, if $a(z)$ at some point were to approach the form of a step function, the $s$ functional would have a term which resembled the stress field to a screw dislocation impinging upon the crack lip al a right angle. Any realistic $a(z)$ that describes a dislocation segment which has popped out will contain two regions of comparatively large slope, and the perturbations of the stress field due to the two individual zones reinforce each other, with the net result that the right-hand side of (37) is sufficiently modified by this effect to cause a solution, otherwise being of order $2 \varepsilon$, to be increased. This shape is consistent with the shallow loop proposed by HAASEN (1983) and discussed by SCHÖCK and Püschl (1991).

TABLE 3. Activation energies: $\mathrm{Cu}, \mathrm{Fe}$, and $\mathrm{Si}$ based on $3 \mathrm{D}$ asymptotic calculation

\begin{tabular}{|c|c|c|c|}
\hline$\frac{G}{G_{\text {crit }}}$ & $\frac{\Delta E_{\text {Cu, partial }}}{k T_{\text {room }}}$ & $\frac{\Delta E_{\mathrm{rc}, \text { full disl. }}}{k T_{\text {room }}}$ & $\frac{\Delta E_{\mathrm{si}, \text { partal }}}{k T_{\text {room }}}$ \\
\hline 0.1 & 39.4 & 303.2 & 171.0 \\
\hline 0.2 & 31.4 & 242.3 & 136.7 \\
\hline 0.3 & 25.5 & 196.9 & 111.1 \\
\hline 0.4 & 20.6 & 158.8 & 89.5 \\
\hline 0.5 & 16.3 & 125.4 & 70.7 \\
\hline 0.6 & 12.4 & 95.5 & 53.9 \\
\hline 0.7 & 8.87 & 68.4 & 38.6 \\
\hline 0.8 & 5.65 & 43.6 & 24.6 \\
\hline 0.9 & 2.68 & 20.7 & 11.7 \\
\hline 1.0 & 0 & 0 & 0 \\
\hline
\end{tabular}


Contour plots, i.e. plots of constant $\delta$ in $x-z$ space, are shown in Fig. 8 for the particular saddle-point distributions corresponding to the various $\varepsilon$ mentioned above. Since the maximum of the slip $\delta_{\text {tip }}$ at the crack tip is $b / 2-\varepsilon+a(0)$, and is expected to be less than $b$ in a more exact analysis, solutions with $2-3 \varepsilon$ greater than about $b / 2$ (i.e. $\varepsilon / b>0.2$ ) may push the limits of the $3 \mathrm{D}$ asymptotic analysis, although the $2 \mathrm{D}$ analysis remains acceptable for $\varepsilon / b$ up to nearly 0.5 . These problems are seen by the fact that the contour lines exceed $\delta=b$ in Figs 8(c) and (d). Since the misfit energy $\Phi(\delta)$ has been expanded about the slips of the $2 \mathrm{D}$ minimum energy state, there is nothing in the asymptotic formulation to reveal that $\delta \approx b$ should be an upper limit to the slip. It is possible that the $\Delta E$ values are, nevertheless, approximately correct even when the local slips of the asymptotic analysis exceed $b$, but such must be checked by further work. The fact that the results at loadings much reduced from critical remain in the range of 5-10 $\Delta U_{\mathrm{acl}}^{2 \mathrm{D}}$ suggests some degree of continued validity.

(a)

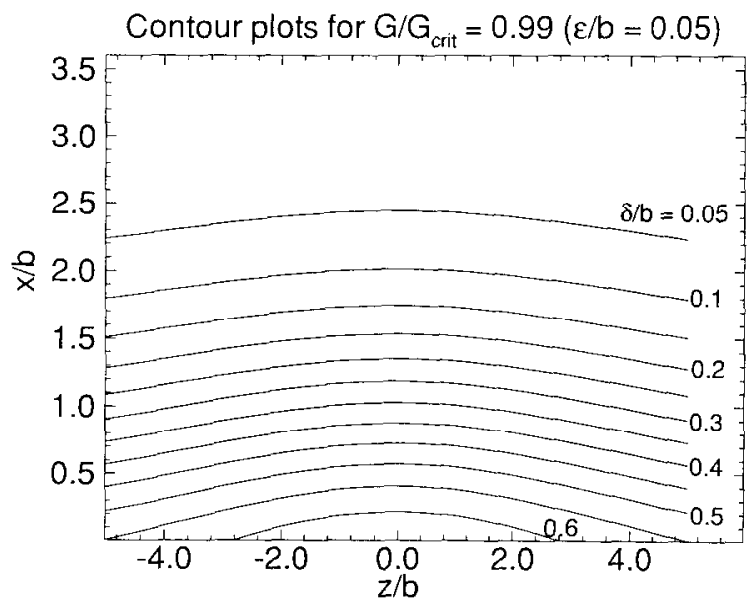

(b)

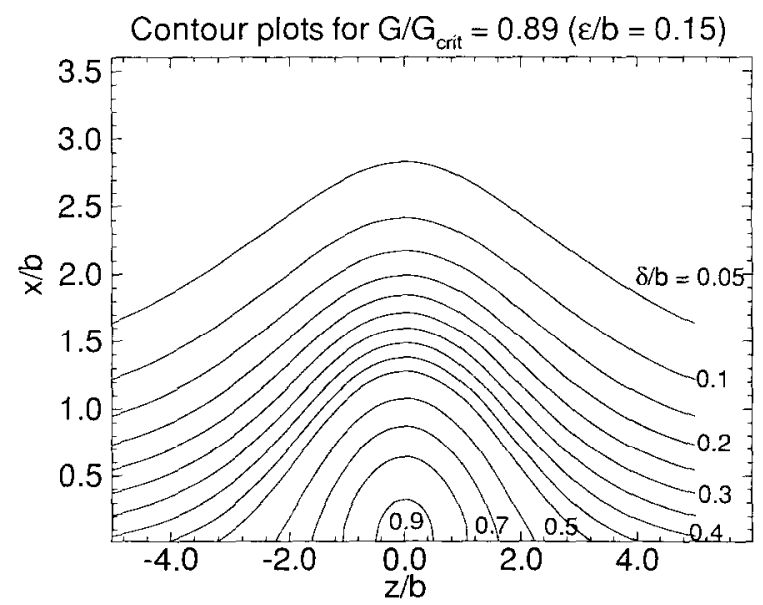

FIG. 8. Contour plots of the 3D slip distribution for: (a) $G / G_{\text {crit }}=0.99(\varepsilon / b=0.05)$, (b) $G / G_{\text {crit }}=0.89$ $(\varepsilon / b=0.15)$, (c) $G / G_{\text {crit }}-0.71(\varepsilon / b-0.25)$, and (d) $G / G_{\text {crit }}=0.25(c / b=0.45)$. 
(c)

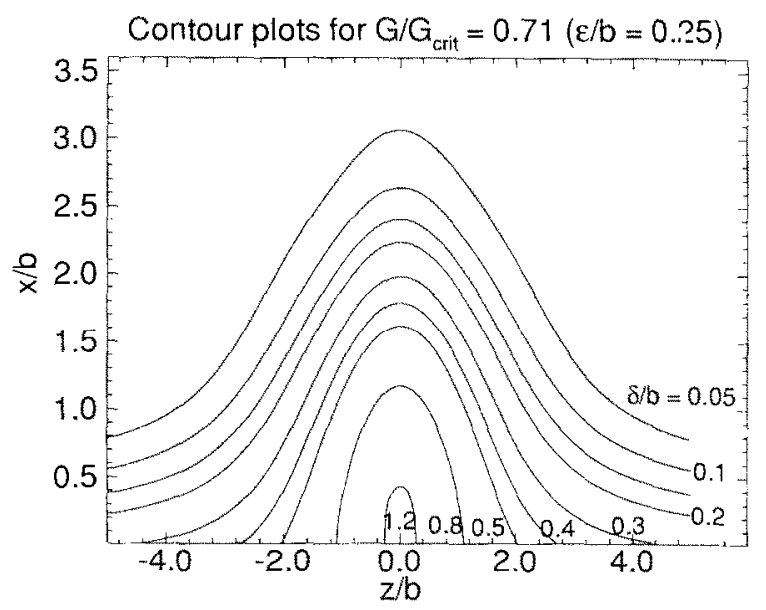

(d)

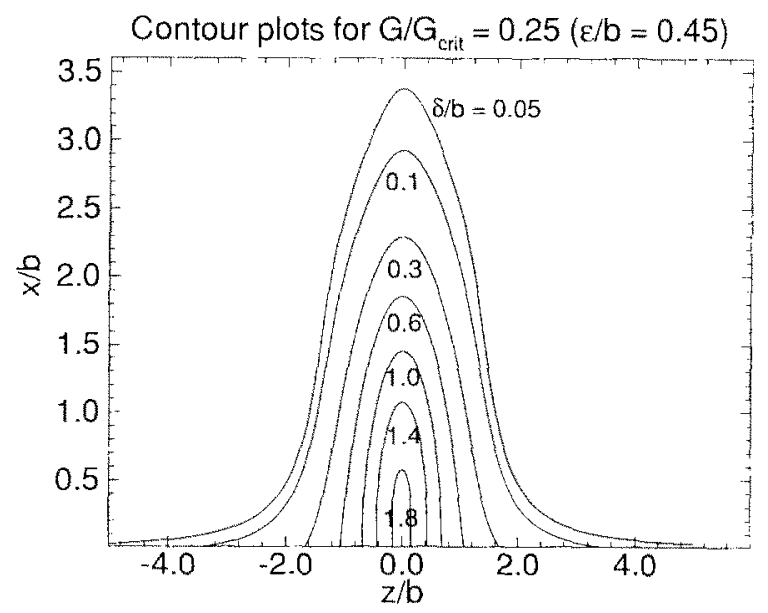

FiG. 8. Continuted.

\section{Implications for Dislocation Nucleation at Elevated Temperaturfs}

\section{Dislocation nucleation in metals}

An elementary calculation of a $\Delta E / k T$ below which there is spontaneous nucleation now follows. Since dislocation nucleation is considered to be a thermally activated proceess, at finite temperatures a dislocation segment may, with the aid of thermal fluctuations, be nucleated under a stress level that would not be sufficient for "instantaneous" nucleation, or for nucleation at $0 \mathrm{~K}$. A given dislocation segment must "wait" until a thermal fluctuation is successful in inducing emission, and the net result is that the total nucleation rate of dislocations must scale with an "attempt" frequency, here taken as $\left(c_{\text {shear }} / b\right)$, where $c_{\text {shear }} \approx 3 \mathrm{~km} / \mathrm{s}$ is the shear wave speed, multiplied by the Boltzmann probability that an incipient dislocation loop possesses an energy greater or equal to the activation energy $\Delta E$, thus giving an Arhennius-type relation. 
Similar arguments are given by SсHӧск (1980) for thermally activated dislocation motion via the nucleation and propagation of a double kink and MATTHEWs et al. (1976) for the nucleation of dislocations at a free surface. Thus

$$
D=n\left(c_{\text {shear }} / b\right) \exp (-\Delta E / k T)
$$

where $v$ is interpreted as the frequency of spontaneous nucleation events per unit distance along the crack front, $n$ is taken as the number of nucleation sites per unit length of crack front, taken here as $n=1 / 10 h$, where $10 b$ is used in light of the broad shape functions shown in Fig. 6. Assuming that $v \approx 10^{6} / \mathrm{s} \mathrm{mm} \mathrm{describes} \mathrm{spontaneous}$ nucleation on a laboratory time scale for a metal, solution of (39) gives a borderline $\Delta E / k T \approx 30$.

Examination of Table 3 (the 3D results) leads to the conclusion that thermal activation would be sufficient (at room temperature) to spontaneously emit a partial dislocation in $\mathrm{Cu}$ at loadings of $G \approx 0.2 G_{\text {crii }}$ or greater, and a full dislocation in $\mathrm{Fe}$ at loadings of $G \approx(0.8-0.9) G_{\text {crit }}$ or greater, keeping in mind that $G_{\text {crit }}$ is the critical loading for dislocation nucleation without help from thermal activation. At $T=2 T_{\text {room }}$, these values for spontaneous nucleation would, e.g., change to approximately $<0.1 G_{\text {crit }}$ for $\mathrm{Cu}$ and $(0.7-0.8) G_{\text {crit }}$ for $\mathrm{Fe}$. The relatively low value of the activation energy for $\mathrm{Cu}$ lcads to the conclusion that dislocation nuclcation is remarkably casy at room temperature, i.e. an energy release rate of about $20 \%$ of the value at $T=0$ is required. It is perhaps best, however, to think in terms of the applied stress intensity factor, which varies linearly with an applied stress. If $K_{\mathrm{II}}^{\text {cit }}$ is defined as the critical stress intensity for nucleation, then at room temperature a dislocation in $\mathrm{Cu}$ would spontaneously emit at approximately $\sqrt{0.2} K_{\mathrm{II}}^{\text {crit }} \approx 0.45 K_{\mathrm{II}}^{\text {crit }}$.

\section{Dislocation nucleation in silicon}

The borderline $\Delta E / k T$ should be re-calculated for the case of $\mathrm{Si}$ in light of experimental observations which show crack tip shielding occurring at or above the transition temperature with as few as five dislocations (CHIAO and CLARKE, 1989) but more commonly on the order of magnitude of 100 (SAMUels and RoBERTS, 1989; WARREN, 1989), on time scales comparable to a minute. Although no nucleation rate has been specifically measured in the experimental work referenced, an approximation would be to assume that $1 / \mathrm{s} \mathrm{mm}$ describes nucleation under the conditions noted. Due to the lngarithmic dependency of $\Lambda F / k T$ on 12 as predicted by (39), an order of magnitude difference in the assumed nucleation rate does not appreciably affect the calculation. The solution of (39), using the Burgers vector of a Shockley partial in $\mathrm{Si}$, and $v=1 / \mathrm{s} \mathrm{mm}$ gives $\Delta E / k T \approx 43$. Using similar reasoning, MATTHEWs el al. (1976) give a similarly-defined cutoff value of $\Delta E / k T \approx 50$, which is discussed in further detail by FITZGERALD et al. (1989) in connection with the nucleation of dislocations at a free surface in $\mathrm{Si}$.

Examination of Tables 1 and 3 leads to the conclusion that a dislocation would be spontaneously emitted in $\mathrm{Si}$ when $G \approx 0.6-0.7 G_{c r i t}$ at room temperature, and at $G \approx 0.4 G_{\text {crit }}$ at twice room temperature. An important issue is that of whether $G$ can be arbitrarily increased to the critical value before cleavage occurs. This consideration is especially important in the case of silicon, which cleaves at room temperature, i.e. 
as $G$ is increased, its critical value for cleavage (usually approximated as the Griffith energy) is attained before conditions for dislocation nucleation prevail. The activation energy results may be used to determine the temperature at which it is possible to nucleate a dislocation when the material is loaded to the Griffith load. By making use of this discriminating condition, it is possible to predict a brittle-to-ductile transition temperature based on dislocation nucleation. The details of this type of calculation are the subject of an upcoming paper (BELTZ and RICE, 1994), and only the highlights are given here. in part because the present work neglects tension-shear coupling which is thought to be important for nucleation in $\mathrm{Si}$.

In order to proceed with the calculation, it is necessary to consider a particular geometry. Since Si cleaves along its $\{111\}$ planes, it makes sense to consider the competition between cleavage and dislocation nucleation on another inclined $\{111\}$ slip plane. For example, consider a crack on a (111) plane with its tip along the [0]1] direction (see Fig. 9). If a mode I load is imposed, the favored slip plane for nucleation is the (T11) plane which is inclined at 70.53 with respect to the fracture plane. The critical load for nucleation of a Shockley partial at $0 \mathrm{~K}$ on this slip plane is approximately $5.29 \gamma_{\text {us }} \approx 10.1 \mathrm{~J} / \mathrm{m}^{2}$, where $\gamma_{\text {ui }}$ has been taken here as the relaxed value calculated by KaXIRAS and DUESBERY (1993). This result depends on many factors, such as the coupling between tension and shear across the slip plane, and the orientation of the Burgers vector; these issues and their effect upon the activation energy are addressed in further detail by Sun et al. (1993) and BelTZ and RICE (1994). The Griffith load is laken here as $3.12 \mathrm{~J} / \mathrm{m}^{2}$. which is based on the TYSON (1975) correlation with formation energy and also happens to be the value calculated quantum mechanically by HuANG et al. (1989) for a reconstructed $\{111\}$ surface using density. functional theory, i.e. the same method used by KAXIRAS and DUESBERY (1993) to calculate the unstable stacking energy that goes into $G_{\text {crit }}$. At the Griffith load, $G / G_{\text {сті }}=0.31$, corresponding to an activation energy of $(0.53) \mu h^{3} /\left(1-1^{\prime}\right) \approx 4.47 \times$. $10^{19} \mathrm{~J} / \mathrm{m}^{2} \approx 2.8 \mathrm{eV}$, based on the asymptotic $3 \mathrm{D}$ calculation (on which the numbers in Table 3 are based). Using the discriminating condition of $\Delta E / k T=43$ discussed above, the transition temperature turns out to be $T_{0}=480 \mathrm{C}$. This preliminary calculation underestimates the actual transition temperature range [c.g. the value of $562 \mathrm{C}$ at a strain rate of $1.3 \times 10^{\circ}$ mentioned earlier in this paper, from work by SAMUELs and RoBERTS (1989)]. The calculation is consistent, however.

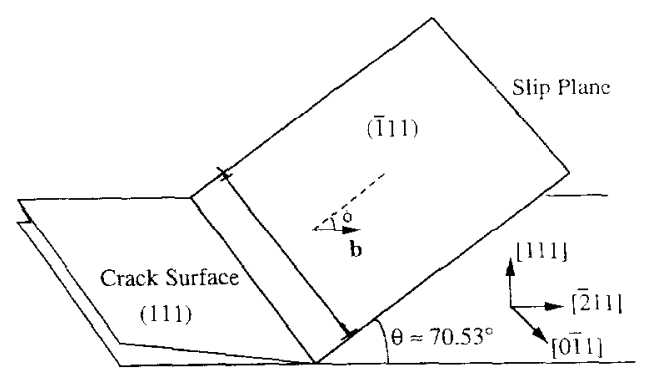

F1G. 9. Schematic depicting the geometry used for the analysis of thermally activated dislocation nucleation in silicon. 
with the idea that thermally activated dislocation nucleation is at least a necessary requirement for the brittle-to-ductile transition to occur, while the transition itself may be due to the strong temperature dependence of dislocation mobility in Si. Dislocations, once nucleated, must still be swept away from the crack tip to relax stress.

\section{Remarks on the activation energy for molecular dynamics (MD) simulations}

In MD simulations of crack tips, it is typical to devise atomic arrays which extend many atom spacings in the $x$ - and $y$-directions, i.e. in the plane perpendicular to the crack tip, but only over a small number of atomic planes (as few as two in simulations of cracks in fec or bec lattices) in the $z$-direction. The Newtonian dynamical equations for the atoms are solved by assuming that atom locations in the layer considered are repeated periodically in the $z$-direction. Let $H$ be the layer thickness. The layer itself, with appropriate modification of atomic force laws in a way that would correspond to periodic repeats of its atom positions, may be considered as a dynamical system to which the ideas of statistical mechanics may be applied. For a thin layer, the imposed periodicity will force any shear distribution at the crack tip to be $2 \mathrm{D}$ in character. Thus if $\Delta E_{\mathrm{icl}}^{\mathrm{MD}}=\Delta E_{\mathrm{icl}}^{\mathrm{MD}}(H)$ is the activation energy for a MD model involving thickness $H$ of the periodically repeated layer, then for a thin layer, $\Delta E_{\text {act }}^{\mathrm{MD}} \approx H \Delta U_{\mathrm{act}}^{2 \mathrm{D}}$. Here $\Delta U_{\text {ict }}^{2 \mathrm{D}}$ is the $2 \mathrm{D}$ activation energy per unit length, which has been calculated here based on the Peierls model and is given in Fig. 4 and Table 1. Only with increasing $H$ can shear configurations develop that are localized along the crack front, like in Figs 6 and 8 , so that we may expect $\Delta E_{i k 1}^{\mathrm{Mu}}$ to increase initially as $H \Delta U_{\text {act }}^{2 \mathrm{D}}$ but to ultimately saturate at $\Delta E_{\mathrm{ac}}$, the $3 \mathrm{D}$ activation energy, as $H$ becomes large.

From Fig. 7 and Tables 1 and 3, we predict that $H \Delta U_{\mathrm{act}}^{2 \mathrm{D}}$ will generally be smaller than $\Delta E_{\text {act }}$ unless $H$ exceeds $5-10 b$. For loadings that are near to critical, which are the cases most feasible for MD study of activated emission because of the computational time steps involved, and which are also the cases for which our calculations of $\Delta E_{\mathrm{act}}$ are thought to be most reliable, $H \Delta U_{\mathrm{act}}^{2 \mathrm{D}}$ will be smaller than $\Delta E_{\mathrm{act}}^{\mathrm{u}}$ unless $H$ exceeds about $17 \mathrm{~b}$. Thus the predicted activation energy to correspond with a MD simulation will vary with layer thickness $H$ roughly as shown in Fig. 10 ; the solid curve is a guess, but it must have the small and large $H$ behavior shown by the dashed lines. Figure 10 has been drawn for a loading $G \approx 0.7 G_{\text {crit }}$, at which (Fig. 7) $\Delta E_{\mathrm{acl}} \approx 10 \mathrm{~b} \Delta U_{\mathrm{act}}^{2 \mathrm{I}}$, so that the dashed lines meet at $H=10 \mathrm{~b}$. The lines meet at yet larger $H$ values, as large as $H \approx 17 b$, for higher load levels like $G \approx 0.9 G_{\text {crit }}$, and meet at smaller $H$ values at lower load levels, perhaps as small as $H \approx 5 b$ if our asymptotic predictions continue to be approximately correct at low load levels.

As an example, consider a crack on the (100) plane, growing in the [011] direction, in a bcc lattice. For the usual $\langle 111\rangle$ bcc slip direction, $b=(\sqrt{3} / 2) a_{0}$, where $a_{0}$ is the side length of a unit cube in the lattice. The lattice geometry allows consideration of a minimum thickness $H_{\text {min }}$ of the simulated layer that includes just two (0I1) atomic planes, in which case the periodic repeat distance is the diagonal of a cube face, $H_{\mathrm{m} \text { in }}=$ $\sqrt{2} a_{0}$. Thus $H_{\min } / b=2 \sqrt{2} / \sqrt{3} \approx 1.6$. For such an MD model, and for the loading $G \approx 0.7 G_{\text {crit }}$ considered in Fig. 10, we have $\Delta E_{\text {alct }}^{\mathrm{MD}} \approx 0.16 \Delta E_{\text {act }}$. Then, $T=100 \mathrm{~K}$ in the MD simulation of the two-atom-plane layer should give thermal activation effects 


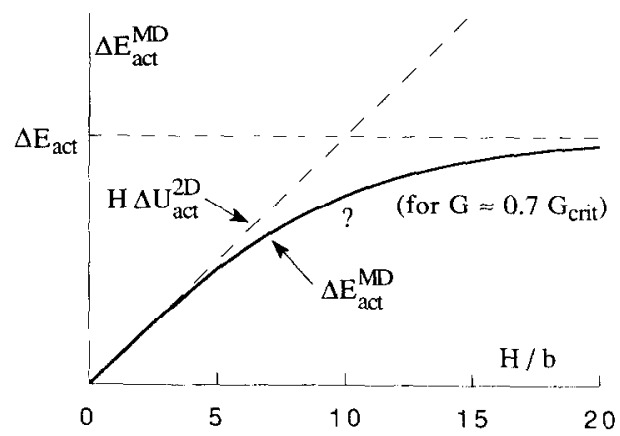

Fig. 10. Activation energy $\Delta E_{\text {act }}^{\mathrm{MD}}$ (solid line, exact shape unknown) corresponding to molecular dynamics simulation of a layer of atomic planes, occupying thickness $H$ in the direction parallel to the crack tip, with periodic conditions at the layer faces. Here $\Delta E_{\text {act }}$ is the $3 \mathrm{D}$ activation energy $\Delta E$ of Fig. 7 . Drawn for loading $G \approx 0.7 G_{\text {crii }}$; value of $H$ at which dashed lines intersect increases with $G / G_{\text {crit }}$.

similar to $T \approx 600 \mathrm{~K}$ for the $3 \mathrm{D}$ solid. For $G \approx 0.9 G_{\mathrm{crit}}, \Delta E_{\mathrm{act}}^{\mathrm{MD}} \approx 0.09 \Delta E_{\mathrm{atc}}$, and $T=100 \mathrm{~K}$ in the MD simulation should then give effects similar to $T \approx 1000 \mathrm{~K}$ for the $3 \mathrm{D}$ solid. Hence the MD model will greatly overestimate the already large effects of temperature that we have predicted based on $\Delta E_{\mathrm{act}}$. These are important effects to consider when planning and interpreting MD simulations.

\section{SUMmary}

The activation energy for dislocation nucleation from a crack tip has been calculated within the Peierls framework. The advantage of this method is that incipient dislocations are treated as a gradual build-up of a tomic shear across a slip plane, thereby eliminating uncertainties associated with analyzing fully formed dislocations near a crack tip. Furthermore, the saddle point associated with unstable dislocation emission is treated in a similar manner. The activation energy per unit length, a $2 \mathrm{D}$ calculation, is calculated exactly as well as asymptotically for loads near the critical load for emission. The activation energy for emission of a 3D loop is also calculated asymptotically. It is found that a good approximation of the 3D result generally consists of multiplying the $2 \mathrm{D}$ result by $5-10 \mathrm{~b}$ (but by as much as $17 \mathrm{~b}$ for loadings very near to critical). Specific values of the activation energy for $\mathrm{Cu}, \mathrm{Fe}$, and $\mathrm{Si}$ are calculated, and the results are used to rationalize the spontaneous emission of dislocations due to thermal effects at loads below the critical load. A preliminary calculation of the activation energy to nucleate a (glide set) Shockley partial in silicon (based on the 3D asymptotic approach) when loaded to the (iriffith cleavage load indicates that thermal activation of dislocations is possible at the observed transition temperatures. For the future, it is important to devise a rigorous but non-asymptotic $3 \mathrm{D}$ calculation of $\Delta E$, and also to better understand inherent limits to the Peierls framework for modeling dislocation nucleation, e.g., due to its neglect of the energy of surface exposed at the crack tip by dislocation blunting. 


\section{ACKNOWLEDGEMENTS}

This research was supported by grants N00014-92-J-1960 and N00014-90-J-1379 from the ONR Mechanics Division to Harvard University, and by a University Research Initiative (subcontract POAVB38639-0 to Harvard from the University of California, Santa Barbara, based on ONR/DARPA contract N00014-86-K-0753). Integral equations in connection with the 3D calculations were solved under NSF support on a Cray Y-MP at the Pittsburgh Supercomputing Center. Insightful discussions with Drs L. B. Freund, A. S. Argon, and R. Thomson are gratefully acknowledged. GEB also acknowledges the support of a postdoctoral appointment with the Solid Mechanics group at Brown University during the final stages of the work.

\section{REFERENCES}

Anderson, P. M. (1986) Ductile versus brittle crack tip response. Ph.D. thesis, Harvard University, Cambridge, MA.

ANDERSON, P. M. and Rice, J. R. (1986) Dislocation emission from cracks in crystals or along crystal interfaces. Scripta Metall. 20, 1467-1472.

ArGon, A. S. (1987) Brittle to ductile transition in cleavage fracture. Acta Metall. 35, 185196.

BeLtz, G. E. (1992) The mechanics of dislocation nucleation at a crack tip. Ph.D. thesis, Harvard University, Cambridge, MA.

BeLTL, G. E. and Rice, J. R. (1991) Dislocation nucleation versus cleavage decohesion at crack tips. Modeling the Deformation of Crystalline Solids: Physical Theory, Application, and Experimental Comparisons (ed. T. C. Lowe, A. D. Rollett, P. S. Follansbee and

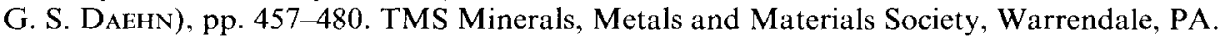

Beltz, G. E. and Rice, J. R. (1992) Dislocation nucleation at metal/ceramic interfaces. Acta Metall. 40, S321-S331.

BeLtz, G. E. and RICE, J. R. (1994) The activation energy for dislocation nucleation in silicon. Manuscript in preparation.

Brede, M. and HaAsen, P. (1988) The brittle-to-ductile transition in doped silicon as a model substance. Acta Metall. 36, 2003-2018.

Chiao, Y.-H. and Clarke, D. R. (1989) Direct observation of dislocation emission from crack tips in silicon at high temperatures. Acta Metall. 37, 203-219.

Duesbery, M. S., Michel, D. J., KaXiras, E. and Joos, B. (1991) Molecular dynamics studies of defects in Si. Defects in Materials (ed. P. D. Bristowe, J. E. Epperson, J. E. Griffith and Z. Liliental-Weber), Materials Research Society, Vol. 209, pp. 125-130.

Erdogan, F. (1975) Complex function technique. Continuum Physics (ed. A. C. Eringen), pp. 523 603. Academic Press, New York.

ERdogan, F. and GuPTA, G. D. (1972) On the numerical solution of singular integral equations. Q. Appl. Math. 29, 525-534.

Fitzgerald, E. A., Watson, G. P., Proano, R. E., Ast, D. G., Kirchnir, P. D., Pfttit, G. D. and WoOdall, J. M. (1989) Nucleation mechanisms and the elimination of misfit dislocations at mismatched interfaces by reduction in growth area. J. Appl. Phys. 65, 22202237.

GAo, H. (1988) Applications of 3D weight functions in elastic crack analysis. Ph.D. thesis, Harvard University, Cambridge, MA.

GAO, H. and RICE, J. R. (1989) Application of 3D weight functions - II. The stress field and energy of a shear dislocation loop at a crack tip. J. Mech. Phys. Solids 37, 155-174.

HaAsen, P. (1983) Electronic processes at dislocation cores and crack tips. Atomistics of Fracture (ed. R. M. LATANision and J. R. PICKENS), NATO Advanced Research Institute Proceedings, pp. 707-730. Plenum Press, New York. 
Hirsch, P. B. and Roberts, S. G. (1991) The brittle ductile transition in silicon. Phil. Mag. A64, 55-80.

Hirsch, P. B., Samuels, J. and Roberts, S. G. (1989) The brittle-ductile transition in silicon. II. Interpretation. Proc. R. Soc. A421, 25-53.

HSIA, K. J. and ARGON, A. S. (1993) Experimental study of micromechanisms of brittle-toductile transition in Si single crystals. Submitted to Mater. Sci. Engng A.

Huang, Y. M., Sprencl, J. C. H., Sankey, O. F. and Adams, G. B. (1991) The influence of internal surfaces on the $(2 \times 1)$ surface shuffle and glide cleavage reconstructions for Si (111). Surf. Sci. 256, 344-353.

KaXIRAS, E. and Dulsbery, M. S. (1993) rree energies of generalized stacking faults in Si and implications for the brittle-ductile transition. Phys. Rev. Lett. 70, 3752-3755.

Li, J. C. M. (1981) Dislocation sources. Dislocation Modelling of Physical Systems (ed. M. F. Ashisy, R. Bullough, C. S. Hartli:y and J. P. Hirth), Acla-Scripta Metallurgica Conference Procecdings, pp. 498-518. Pcrgamon Press, Oxford.

LI, J. C. M. (1986) Computer simulation of distocations cmilted from a crack. Scripta Metall. $20,1477-1482$.

Mason, D. (1979) Segregation-induced embrittlement of grain boundaries. Phil. Mag. 39, 455 468.

Matthews, J. W., Blakeslele, A. E. and Mader, S. (1976) Use of misfit strain to remove dislocations from epitaxial thin films. Thin Solid Films 33, 253-266.

Michor, G. and George, A. (1986) Dislocation emission from cracks--observations by Xray topography in silicon. Scripta Metall. 20, 1485-1500.

Rice, J. R. (1992) Dislocation nucleation from a crack tip: an analysis based on the Pcierls concept. J. Mech. Phys. Solids 40, 239-271.

Rick. J. R., Bist\%, G. E. and Sun. Y. (1992) Peierls framework for dislocation nucleation from a crack tip. Topics in Fracture and Fatigue (ed. A. S. Akgon), Chap. 1, pp. 1-58. Springer, Berlin.

Rick, J. R. and Thomson, R. (1974) Ductile versus brittle behavior of crystals. Phil. Mag. 29, $73-97$.

Samul:Ls, J. and Roberts, S. G. (1989) The brittle ductile transition in silicon. I. Experiments. Proc. R. Soc. A421, 1-23.

Scнöck, G. (1980) Thermodynamics and thermal activation of dislocations. Dislocutions in Solids (ed. F. R. N. Nabarro), Vol. 3, pp. 63-163. North-Holland, Amsterdam.

Scнöck, G. (1991) Dislocation emission from crack tips. Phil. Mag. A63, 111- 120.

Sснӧск, G. and Püschl, W. (1991) The formation of dislocation loops at crack tips in three dimensions. Phil. Mag. A64, 931 -949.

Sun, Y. (1993) Atomistic aspects of dislocation/crack tip interaction. Ph.D. thesis, Harvard University, Cambridge, MA.

SUN. Y., BtLI/, G. E. and Ricr, J. R. (1993) Estinates from atomic models of tension-shear coupling in dislocation nucleation from a crack tip. Mater. Sci. Engng $A$ (in press).

SUN, Y.. Rick, J. R. and TRuSkinovsky, L. (1991) Dislocation nucleation versus cleavage in $\mathrm{Ni}_{3} \mathrm{\Lambda l}$ and $\mathrm{Ni}$. High-temperature Ordered Intermetallic Alloys (ed. L. A. Johnson. D. T. Pole and J, O. Stilgler), Materials Research Society, Vol. 213, pp. 243-248.

Thomson, R. (1986) Physics of fracture. Solid State Physics (ed. H. Ehrinraich and D. Turnbuli.). Vol. 3, pp. 1 129. Academic Press, New York.

Tyson, W. R. (1975) Surface energies of solid metals. Can. Metall. Q. 14, 307-314.

Warres, P. D. (1989) The brittle-ductile transition in silicon: the influence of pre-existing dislocation arrangements. Seripta Metall. 23, 637-642.

\section{Appendix: Numerical Procedure}

The functional $s[\delta(x, z)]$ appearing in the integrand of (37) may be written as (GAO. 1988; GAO and RICI, 1989 ; H. GAO, private communication, 1991) 


$$
\begin{array}{r}
s[\delta(x, z)]=\frac{\mu x}{4 \pi(1-v)} \int_{-\infty}^{\infty} \frac{\delta\left(0^{+}, \tilde{z}\right)-\delta\left(0^{+}, z\right)}{\left[x^{2}+(z-\tilde{z})^{2}\right]^{3 / 2}} \mathrm{~d} \tilde{z}-\int_{x}^{x} \int_{0}^{x} H(x, \tilde{x}, z-\tilde{z})\left[\delta(\tilde{x}, \tilde{z})-\delta\left(0^{+}, z\right)\right] \mathrm{d} \tilde{x} \mathrm{~d} \tilde{z} \\
\\
\left.-\frac{\mu}{4 \pi(1-v)} \int_{-\infty}^{\infty} \int_{0^{+}}^{x}\left\{\frac{\tilde{x}-x}{D^{3}} \frac{\partial}{\partial \tilde{x}}+(1-v) \frac{\tilde{z}-z}{D^{3}} \frac{\partial}{\partial \tilde{z}}\right\} \delta(\tilde{x}, \tilde{z}) \mathrm{d} \tilde{x} \mathrm{~d} \tilde{z}, \quad \text { (A } 1\right)
\end{array}
$$

where

$$
H \equiv \frac{\mu}{2 \pi^{2}(1-v)}\left\{\left[1+\frac{2 v}{2-v} \frac{\partial}{\partial \bar{z}}(\tilde{z}-z)\right] F+\begin{array}{cc}
v^{2} & \partial^{2} G \\
2-v & \partial \bar{z} \partial z
\end{array}\right\}
$$

and

$$
\begin{aligned}
F(x, \tilde{x}, z-\tilde{z}) & =\frac{1}{D^{3}}\left\{\frac{D}{2 \sqrt{x} \tilde{\tilde{x}}}-\arctan \frac{D}{2 \sqrt{x \tilde{x}}}\right\}, \\
G(x, \tilde{x}, z-\tilde{z}) & =\frac{1}{D} \arctan -\frac{D}{2 \sqrt{x} \tilde{x}}, \\
D^{2} & =(x-\tilde{x})^{2}+(z-\tilde{z})^{2} .
\end{aligned}
$$

Replacing $\delta(x, z)$ with $a(z) g(x)$, formulating the quantity $\{a(z) s[g(x)]-s[a(z) g(x)]\}$, and rearranging [noting that $g(0)$ is unity] gives

$$
\begin{aligned}
\{a(z) s[g(x)]-s[a(z) g(x)]\}= & -\frac{\mu x}{4 \pi(1-v)} \int_{-x}^{x} \frac{a(\tilde{z})-a(z)}{\left[x^{2}+(z-\tilde{z})^{2}\right]^{3 / 2}} \mathrm{~d} \tilde{z} \\
& +\int_{x}^{x} \int_{0}^{x} H(x, \tilde{x}, z-\tilde{z}) g(\tilde{x})[a(\tilde{z})-a(z)] \mathrm{d} \tilde{x} \mathrm{~d} \tilde{z} \\
& +\frac{\mu}{4 \pi(1-v)} \int_{-\infty}^{x} \int_{0^{+}}^{x} \frac{(\tilde{x}-x) g^{\prime}(\tilde{x})[a(\tilde{z})-a(z)]}{D^{3}} \mathrm{~d} \tilde{x} \mathrm{~d} \tilde{z} \\
& +\frac{\mu}{4 \pi} \int_{-\infty}^{\infty} \int_{0^{+}}^{\infty} \frac{(\tilde{z}-z) g(\tilde{x}) a^{\prime}(\tilde{z})}{D^{3}} \mathrm{~d} \tilde{x} \mathrm{~d} \tilde{z},
\end{aligned}
$$

where the "prime" notation on functions $a$ and $g$ refers to differentiation with respect to the argument. Multiplying (A4) by $g(x)$ and integrating gives the right-hand side of (37),

$$
\int_{0}^{x_{1}}\{a(z) s[g(x)]-s[a(z) g(x)]\} g(x) \mathrm{d} x=\int_{x}^{x}[a(\tilde{z})-a(z)] F_{\mathrm{A}}(z-\tilde{z}) \mathrm{d} \tilde{z}+\int_{-\infty}^{\infty} a^{\prime}(\tilde{z}) F_{\mathrm{B}}(z-\tilde{z}) \mathrm{d} \tilde{z},
$$

wherc

$$
\begin{aligned}
& F_{\mathrm{A}} \equiv-\frac{\mu}{4 \pi(1-v)} \int_{0}^{\infty} \frac{x g(x)}{\left[x^{2}+\frac{(z-\bar{z})^{2}}{3 / 2}\right.} \mathrm{d} x+\int_{0}^{\infty} \int_{0}^{\infty} H(x, \tilde{x}, z-\tilde{z}) g(\tilde{x}) g(x) \mathrm{d} \tilde{x} \mathrm{~d} x \\
& +\frac{\mu}{4 \pi(1-v)} \int_{0}^{\infty} \int_{0}^{\infty} \frac{(\tilde{x}-x) g^{\prime}(\tilde{x}) g(x)}{D^{3}} \mathrm{~d} \tilde{x} \mathrm{~d} x
\end{aligned}
$$

and

$$
F_{\mathrm{B}}=\frac{\mu}{4 \pi} \int_{0}^{x} \int_{0}^{x} \frac{(\tilde{z}-z)}{D^{3}} \frac{g(\tilde{x})}{g(x)} \mathrm{d} \tilde{x} \mathrm{~d} x
$$

Noting that the integrands in (A6) and (A7) remain finite over all $x$ and $\tilde{x}$ except when $z-\tilde{z}=0$, 
the functions $F_{\mathrm{A}}(z-z)$ and $F_{\mathrm{B}}(z-z)$ are obtained by numerically integrating the representations given in (A6) and (A7) via a 21 -point Gauss $\mathrm{K}$ ronrod rule with a relative error in the result of $10{ }^{4}$. The functions become singular for $z=\Xi$; however, that point is not needed: during the actual solution of (37), the integral given by (A5) is rapidly carricd out via the Gauss Chebyshev integration formula (ERDOGAN and GUPTA, 1972; ERDOGAN, 1975), which never requires the evaluation of the integrands at $z=z$.

To achieve a suitable format in which to use the Gauss-Chebyshev scheme, the integrals in (A5) are rewritten with semi-infinite domains of integration, noting that $F_{A}$ is an even function and $F_{\mathrm{B}}$ is an odd function:

$$
\begin{aligned}
\int_{0}^{x}\{a(z) s[g(x)]-x[a(z) g(x)]\} g(x) \mathrm{d} x \\
\quad=\int_{0}^{x}[a(z)-a(z)]\left\{F_{\mathrm{A}}(z-z)+F_{\mathrm{A}}(z+z)\right\} \mathrm{d} z+\int_{0}^{x} a^{\prime}(z)\left\{F_{\mathrm{B}}(z-z)-F_{\mathrm{B}}(z+z)\right\} \mathrm{d} z
\end{aligned}
$$

Next, the following change of variables is made which maps the semi-infinite domain onto the region $-1<\eta<1$ :

$$
==b \frac{1+\eta}{1-\eta}, \quad z=b \begin{aligned}
& 1+\xi \\
& 1-\xi
\end{aligned}
$$

The domains of $\eta$ and $\xi$ are then discretized and the integral (37) is pointwise enforced at values of $n$ given by

$$
\eta_{i}=\cos \frac{\pi(2 i-1)}{2(n+1)}, \quad i=1, \ldots, n+1
$$

Integration is carried out by discretizing the domain of $\xi$ in the manner

$$
\xi_{i}=\cos i \pi+1, \quad j=1, \ldots n .
$$

The remainder of the numerical procedure proceds cxactly as outlined by BEITz and RICL: (1992), with $n$ taken as 50 . 